\title{
Effective Lagrangian approach to neutrinoless double beta decay and neutrino masses
}

\author{
Francisco del Aguila, ${ }^{1}$ Alberto Aparici, ${ }^{2}$ Subhaditya \\ Bhattacharya, ${ }^{3}$ Arcadi Santamaria ${ }^{2}$, and Jose Wudka ${ }^{3}$ \\ ${ }^{1}$ CAFPE and Departamento de Fisica Teorica y del Cosmos, \\ Universidad de Granada, E-18071 Granada, Spain \\ ${ }^{2}$ Departament de Fisica Teorica, Universitat de Valencia and IFIC, \\ Universitat de Valencia-CSIC, Dr. Moliner 50, \\ E-46100 Burjassot (Valencia), Spain \\ ${ }^{3}$ Department of Physics and Astronomy, \\ University of California, Riverside CA 92521-0413, USA
}

\begin{abstract}
Neutrinoless double beta $(0 \nu \beta \beta)$ decay can in general produce electrons of either chirality, in contrast with the minimal Standard Model (SM) extension with only the addition of the Weinberg operator, which predicts two left-handed electrons in the final state. We classify the lepton number violating (LNV) effective operators with two leptons of either chirality but no quarks, ordered according to the magnitude of their contribution to $0 \nu \beta \beta$ decay. We point out that, for each of the three chirality assignments, $e_{L} e_{L}, e_{L} e_{R}$ and $e_{R} e_{R}$, there is only one LNV operator of the corresponding type to lowest order, and these have dimensions 5, 7 and 9, respectively. Neutrino masses are always induced by these extra operators but can be delayed to one or two loops, depending on the number of RH leptons entering in the operator. Then, the comparison of the $0 \nu \beta \beta$ decay rate and neutrino masses should indicate the effective scenario at work, which confronted with the LHC searches should also eventually decide on the specific model elected by nature. We also list the SM additions generating these operators upon integration of the heavy modes, and discuss simple realistic examples of renormalizable theories for each case.
\end{abstract}




\section{INTRODUCTION}

The remarkable observation of neutrino oscillations (see [1] and [2,3] for recent reviews) provided the first direct evidence of physics beyond the Standard Model (SM), these effects are best explained by providing the neutrinos by small masses and appropriate mixing angles $[4,5]$ (see also [6] for a recent fit). In contrast with the quark sector, however, neutrino masses are not necessarily of the Dirac type, yet oscillation experiments are not sensitive to determine whether neutrino masses are of the Majorana type [7]. Fortunately there is another process, neutrino-less double-beta $(0 \nu \beta \beta)$ decay $([8,9]$ and [10] for a review) that probes this property of the neutrino sector, and has achieved sufficient sensitivity (see [11-13] for recent reviews) to provide interesting constraints on the lepton-number violating (LNV) processes that can produce it. In this paper we will be concerned with general properties of both neutrino masses and $0 \nu \beta \beta$ decay; we will strive to provide modelindependent description of the effects that concern us, yet we will make connection with specific models that provide concrete and important illustrations of the arguments presented.

Since both neutrino masses and $0 \nu \beta \beta$ decay are low energy processes, an effective Lagrangian approach [14-19] is the proper starting point of any model-independent discussion; in it, all virtual new physics (NP) effects are parameterized by the coefficients, $C^{(n)}$, of the corresponding effective operators $\mathcal{O}^{(n)}$. Explicitly,

$$
\mathcal{L}=\mathcal{L}_{S M}+\sum_{n=5}^{\infty} \sum_{i}\left(\frac{C_{i}^{(n)}}{\Lambda^{n-4}} \mathcal{O}_{i}^{(n)}+\text { h.c. }\right)
$$

where $n$ denotes the canonical dimension of the operator, $i$ labels the independent operators of a given order and $\Lambda$ the NP scale. The $\mathcal{O}_{i}^{(n)}$ respect all the local symmetries of the SM, but not necessarily the global ones; when the NP contains several scales $\Lambda>\Lambda^{\prime}>\cdots$ the coefficients $C$ may contain powers of $\Lambda^{\prime} / \Lambda$ (we will assume that the new physics is weakly coupled and decoupling).

Several earlier papers [20-23] have followed this approach, considering, however, only effective interactions that do not involve the SM gauge bosons. Here we consider a different class of theories where the NP does not couple directly to the quark sector ${ }^{1}$, so that the effective interactions involve only leptons and gauge bosons (coupling to the gauge bosons is

\footnotetext{
${ }^{1}$ Operators involving leptons and quarks with no gauge bosons generate neutrino masses at 1 to 4 loops [2326] and may receive enhancements from top Yukawa couplings.
} 
generated whenever the NP is not a SM gauge singlet). We will show that in this case one can provide a simple classification of the effects that concern us in terms of only three operators, each of which can be generated at tree level by different types of NP. In the unitary gauge these operators give the vertices $\nu_{L} \nu_{L}, W e_{R} \nu_{L}$ and $W W e_{R} e_{R}$ and have dimension 5,7 and 9 , respectively.

This allows for three scenarios wherein one of the operators is generated at tree level and the others via loops. A simple example exhaustively considered in the literature has tree-level generated neutrino masses via a high-scale see-saw mechanism [27-30], with effective $W e_{R} \nu_{L}$ and $W W e_{R} e_{R}$ vertices generated radiatively. Here we concentrate on the complementary situations where the other operators are generated at tree level and neutrino masses are generated radiatively. Though we will discuss the three cases separately it is of course possible for the NP to simultaneously contain all of them. Indeed, in specific models, phenomenological constraints might necessitate such complications; this is the case in realistic left-right (L-R) models [31]. However, the interesting result of the analysis we present is that, whatever the complications, they reduce to combinations of the three cases discussed below whenever our assumptions are applicable.

The classification of NP contributions to $0 \nu \beta \beta$ decay is straightforward. The final state involves two electrons of either chirality which may proceed directly from the effective vertex generated by the NP, or from the $W \nu_{L} e_{L} \mathrm{SM}$ vertex. As a result LNV effects contributing to $0 \nu \beta \beta$ decay can be classified according to the chirality of the final leptons. It is remarkable that, as we shall show, there is only one lowest-order operator for each of the three possible chirality assignments exhibiting a notable connection between chirality and dimension: ${ }^{2}$

$$
\begin{array}{ll}
\mathcal{O}^{(5)}=\left(\overline{\tilde{\ell}_{L}} \phi\right)\left(\tilde{\phi}^{\dagger} \ell_{L}\right), & \text { for two LH leptons (LL), } \\
\mathcal{O}^{(7)}=\left(\phi^{\dagger} D^{\mu} \tilde{\phi}\right)\left(\phi^{\dagger} \overline{e_{R}} \gamma_{\mu} \tilde{\ell}_{L}\right), & \text { for one LH lepton and one RH charged lepton (LR), } \\
\mathcal{O}^{(9)}=\overline{e_{R}} e_{R}^{c}\left(\phi^{\dagger} D^{\mu} \tilde{\phi}\right)\left(\phi^{\dagger} D_{\mu} \tilde{\phi}\right), & \text { for two RH charged leptons (RR). }
\end{array}
$$

Here we omitted flavor indices and denote the light scalar isodoublets by $\phi$, the left-handed (LH) lepton isodoublets by $\ell_{L}$, and right-handed $(\mathrm{RH})$ lepton isosinglets by $e_{R}$; we also use $\tilde{\phi}=i \sigma_{2} \phi^{*}$, and $\tilde{\ell}_{L}=i \sigma_{2} \ell_{L}^{c}$. The electric charge is the sum of the third component of isospin

\footnotetext{
${ }^{2}$ Notice that the $\mathcal{O}^{(5)}$ lepton number $(\mathrm{LN})$ assignment is opposite to that of $\mathcal{O}^{(7)}$ and $\mathcal{O}^{(9)}$ in order to preserve the usual convention for $\mathcal{O}^{(5)}$.
} 

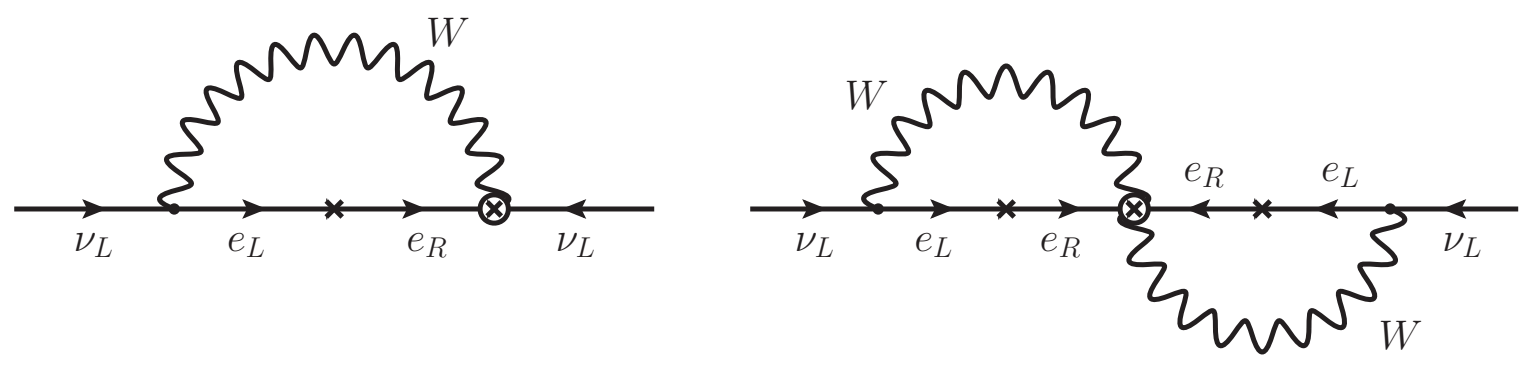

FIG. 1: One (left) and two (right) loop neutrino masses for LR and RR operators, respectively. Arrows indicate fermion number flow.

and the hypercharge, $Q=I_{3}+Y$; thus $\ell_{L}$ and $\tilde{\phi}$ have hypercharge $-1 / 2$, while $\phi$ and $\tilde{\ell}_{L}$ have hypercharge $+1 / 2$.

The operators in Eqs. (2-4) also provide contributions to the neutrino masses; the first one at tree level, as first noted by Weinberg [32] (see also [33]), the other two, radiatively at one and two loops, respectively; see Fig. 1 . We will show that ${ }^{3}$ (here $a, b$ are family indices)

$$
\begin{aligned}
\left(m_{\nu}\right)_{a b} & \propto \frac{v^{2}}{\Lambda} C_{a b}^{(5) *}, & & \text { for LL, } \\
\left(m_{\nu}\right)_{a b} & \propto \frac{v}{16 \pi^{2} \Lambda}\left(m_{a} C_{a b}^{(7)}+m_{b} C_{b a}^{(7)}\right), & & \text { for } \mathrm{LR}, \\
\left(m_{\nu}\right)_{a b} & \propto \frac{1}{\left(16 \pi^{2}\right)^{2} \Lambda} m_{a} C_{a b}^{(9)} m_{b}, & & \text { for RR, }
\end{aligned}
$$

where the vacuum expectation value $(\mathrm{VEV})\langle\phi\rangle \equiv v \simeq 174 \mathrm{GeV}$ is the electroweak symmetry breaking parameter and $m_{a, b}$ the corresponding charged lepton masses. It is worth noting that the contributions from $\mathcal{O}^{(7,9)}$ to $m_{\nu}$ have a natural hierarchy derived from the charged lepton mass factors and may be used to generate textures naturally. We will discuss this issue in a forthcoming publication.

In all cases the neutrino masses vanish when the scale of NP $\Lambda \rightarrow \infty$, as required by the decoupling theorem [34]. This implies that the neutrino oscillation data imposes a upper bound on $\Lambda$, while the limit from $0 \nu \beta \beta$ decay provides a lower bound on this scale. It also follows that the NP cannot be flavor blind, so that specific models in general will be

\footnotetext{
${ }^{3}$ The dominant contributions obtained in specific models are proportional to $\Lambda^{-1}$ since the lowest dimension operator describing neutrino masses is $\mathcal{O}^{(5)}$, and this operator has dimension 5. Once LN is broken, $\mathcal{O}^{(5)}$ will always be generated, at one or two loops (barring model dependent cancellations), even if it does not appear at tree level; this happens in the models we will consider that generate $\mathcal{O}^{(7)}$ or $\mathcal{O}^{(9)}$ at tree level. Deriving these estimates requires care; in particular, estimates obtained using the unitary gauge are often not reliable.
} 
constrained, not only by the neutrino and $0 \nu \beta \beta$ data, but also by lepton-flavor violation (LFV) constraints [35].

If the NP generates any one of these operators at tree level, the remaining two will be generated at one or two loops; when $\mathcal{O}^{(5)}$ is generated radiatively the neutrino masses are proportional to the coefficients of the tree-level generated operator and are, in this sense, predictable. In a companion paper [35] we provide a realistic and highly constrained model that illustrates this scenario, having only $\mathcal{O}^{(9)}$ generated at tree level while $\mathcal{O}^{(7)}, \mathcal{O}^{(5)}$ appear, respectively at one and two loops. In particular, neutrino masses are generated at two loops (see Eq. (7)); the wide literature on radiative neutrino masses provides many additional examples (see for instance [36-39]).

We will see that current data on $0 \nu \beta \beta$ decay implies that the scales associated with NP generating $\mathcal{O}^{(5)}, \mathcal{O}^{(7)}$ and $\mathcal{O}^{(9)}$ at tree level are, respectively $\Lambda>$ $10^{11}\left|C_{e e}^{(5)}\right| \mathrm{TeV}, 10^{2}\left|C_{e e}^{(7)}\right|^{1 / 3} \mathrm{TeV}$, and $\left|C_{e e}^{(9)}\right|^{1 / 5} \mathrm{TeV}$. For first type of NP we expect all collider effects to be negligible; while we expect that the NP responsible for $\mathcal{O}^{(9)}$ will be probed at the LHC [35]. The intermediate case of $\mathcal{O}^{(7)}$ may or may not have collider signatures, depending on the details of the model; we examine one such case below.

In next section we shall define our notation and using the effective Lagrangian approach we classify the lowest order interactions (operators) invariant under the SM gauge group with two external leptons but no quarks, mediating $0 \nu \beta \beta$ decay (it turns out that such operators have equal number of covariant derivatives and $\mathrm{RH}$ leptons). The mechanism for generating neutrino masses is discussed in Section III for the three different cases and compared to the corresponding $0 \nu \beta \beta$ decay amplitude. In Section IV we identify the new particle additions generating those operators at tree level after integrating them out. A more detailed exposition is presented in the appendix. Whereas explicit (renormalizable) models are given for each scenario in Section V. The phenomenological implications for LFV processes and LHC searches are reviewed in Section VI. The last section is devoted to conclusions. As indicated, technical details are collected in the appendix. 


\section{LOWEST ORDER EFFECTIVE OPERATORS CONTRIBUTING TO $0 \nu \beta \beta$ DE- CAY}

The effects of NP below its characteristic scale $\Lambda$ are adequately described by an effective theory involving the light fields and preserving the unbroken local symmetries; the smaller (compared to $\Lambda$ ) the characteristic energy scale of the relevant processes, the higher the accuracy of such a description. This justifies parameterizing $0 \nu \beta \beta$ decay, whose effective scale is some tens of $\mathrm{MeV}$, by a set of effective operators describing $\mathrm{NP}$ above the electroweak scale. These operators must violate $\mathrm{LN}$, as does $0 \nu \beta \beta$ decay and the observed neutrino masses, which we assume to be of Majorana type (we also assume there are no light RH neutrinos).

In this section we will classify the lowest order local operators mediating $0 \nu \beta \beta$ decay. As mentioned in the introduction, this has been addressed previously in the literature, often including quark fields [20-23] (see also [24, 26] for recent models realizing operators with quark fields). In contrast to these papers, we shall assume that the NP does not couple directly to the quark sector, so that all quark interactions are mediated by the electroweak gauge bosons. In the appendix we provide the methodology for constructing the effective operators to any given order, discussing in detail the dominant ones for each final electron chirality.

In order to write down the basis of effective operators we first have to fix the light field content and the symmetries the operators must satisfy. We shall restrict ourselves to the SM fields and local symmetries, although allowing for more that one light scalar doublet, as this gives a few more alternatives and may simplify explicit realistic realizations, as we shall illustrate later on. Operators contributing to $0 \nu \beta \beta$ decay must involve two leptons of either chirality, $\ell_{L}$ or $e_{R}$, and a number of scalar doublets, $\phi$, to make the product invariant under $S U(2)_{L} \times U(1)_{Y}$ transformations. Besides, they can have covariant derivative $\left(D_{\mu}\right)$ insertions, which do not change the field quantum numbers. We will be mainly interested in the lowest order operators of each class, and in the heavy particles whose virtual effects can generate them (assuming only renormalizable couplings).

Before proceeding we note, that in some cases the number of different light scalar doublets $\phi_{i}$ in the theory matters, since for $i>1$ qualitatively new operators become possible; this is related to the appearance of additional physical scalars. When more than one light doublet 
is present one must consider all possible scalar flavor assignments to all allowed operator structures. For example, in the RR case we have an operator of dimension 7, analogous to $\mathcal{O}^{(9)}$, but with no covariant derivatives: $\overline{e_{R}} e_{R}^{c}\left(\phi_{i}^{\dagger} \tilde{\phi}_{j}\right)^{2}$. This field product vanishes if $i=j$ because the scalar product $\phi_{i}^{\dagger} \tilde{\phi}_{j}$ of two isodoublets is antisymmetric; however, if we have two light scalar doublets, the operator does not vanish. Such a theory will contain

light charged scalars $H^{ \pm}$; upon spontaneous symmetry breaking, $\phi_{i}^{\dagger} \tilde{\phi}_{j} \propto H^{-}+\cdots$, so that operator generates the coupling $\overline{e_{R}} e_{R}^{c} H^{-} H^{-}$and will contribute to $0 \nu \beta \beta$ decay if the physical charged Higgs $H^{-}$couples to quarks. Similarly, the number of sets of possible heavy excitations that generate the effective operators after being integrated out increases with the number of independent light scalar doublets.

We will proceed as follows. In the classification of independent operators we will for the most part assume there is only one SM Higgs doublet. We will do the same for the listing of the SM additions (that is, the heavy excitations) generating these operators; in the appendix we also discuss the differences when there is more than one light scalar isodoublet. In the model generating $\mathcal{O}^{(7)}$ below (Section V 2) we make use of two light scalar doublets for illustration, and simplicity; in that particular case the presence of a discrete symmetry guarantees that there is only one lowest-order effective operator involving two leptons, one LH and one RH, despite having two doublets (technically this is because a covariant derivative and a gamma matrix are both required to match chirality and Lorentz indices).

\section{LNV operators with two LH leptons}

The lowest-order operator of this type is the only invariant dimension 5 (Weinberg) operator $[32,33]$ displayed in Eq. (2). The corresponding term in the Lagrangian is

$$
\frac{C_{e e}^{(5)}}{\Lambda} \mathcal{O}_{e e}^{(5)}=\frac{C_{e e}^{(5)}}{\Lambda} \overline{\tilde{\ell}_{e L}} \phi \tilde{\phi}^{\dagger} \ell_{e L} \rightarrow-\frac{v^{2} C_{e e}^{(5)}}{\Lambda} \overline{\nu_{e L}^{c}} \nu_{e L}+\cdots=-\frac{1}{2}\left(m_{\nu}\right)_{e e}^{*} \overline{\nu_{e L}^{c}} \nu_{e L}+\cdots
$$

where the isodoublet corresponds to the first generation (as will be the case throughout this section). In general there are corresponding contributions for all lepton flavors, see Section III, which provide Majorana masses to all the SM neutrinos after electroweak symmetry breaking. This operator contributes to $0 \nu \beta \beta$ decay by transforming the two neutrinos into two LH electrons by the emission of two $W$ gauge bosons (see Fig. 2).

All LNV operators of dimension 6 involve quarks and violate baryon number by \pm 1 unit; 


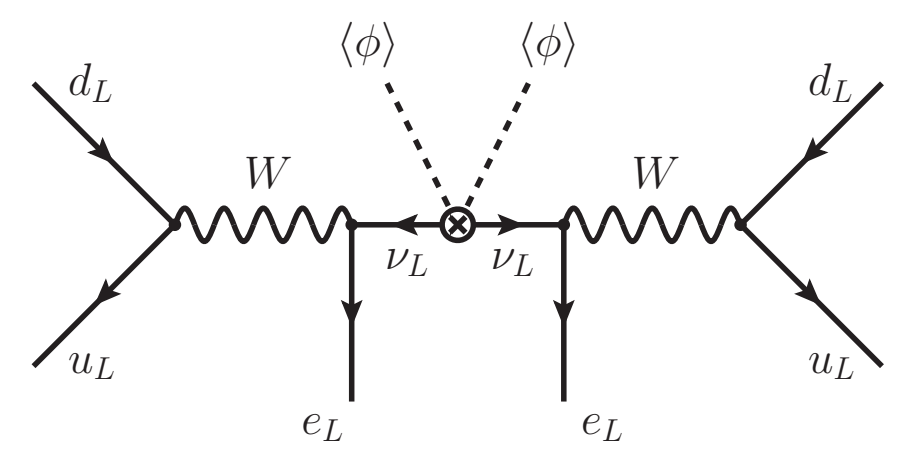

FIG. 2: Tree-level diagram mediated by $\mathcal{O}^{(5)}$ (light neutrino masses) contributing to $0 \nu \beta \beta$ decay.

they also conserve $B-L$ and will not contribute to $0 \nu \beta \beta$ decay. There are, however, three independent operators of dimension 7 that do contribute to $0 \nu \beta \beta$ decay:

$$
\begin{aligned}
\mathcal{O}^{(7-\mathrm{I})} & =\left(\overline{D_{\mu} \ell_{L}} \tilde{\phi}\right)\left(\phi^{\dagger} D^{\mu} \tilde{\ell}_{L}\right), \\
\mathcal{O}^{(7-\mathrm{II})} & =\left(\overline{\ell_{L}} D_{\mu} \tilde{\ell}_{L}\right)\left(\phi^{\dagger} D^{\mu} \tilde{\phi}\right), \\
\mathcal{O}^{(7-\mathrm{III})} & =\left(\overline{\ell_{L}} \tilde{\phi}\right) \partial_{\mu}\left(\phi^{\dagger} D^{\mu} \tilde{\ell}_{L}\right)
\end{aligned}
$$

(see the appendix). One may think that the operators in Eq. (9) generate important contributions to LNV processes; this, however, is not the case. The reason is that any model generating (9) at tree level necessarily also generates $\mathcal{O}^{(5)}$ in Eq. (8) at tree level; all these operators contribute to all the processes we are interested in, but those from (9) will always be suppressed by two additional powers of $\Lambda$ and are subdominant. Accordingly, we will ignore these operators in the phenomenological analysis.

The limit on $0 \nu \beta \beta$ decay, for instance, from ${ }^{76} \mathrm{Ge}[40,41]$ (see also $[11,12]$ for recent reviews) is usually expressed in terms of the electron-electron element of the neutrino mass matrix

$$
\left|\left(m_{\nu}\right)_{e e}\right|<0.24-0.5 \mathrm{eV}
$$

This constraint on $\left(m_{\nu}\right)_{e e}$ is consistent with the neutrino mass limits from oscillations, cosmology and tritium beta decay. When (8) is used to express $\left(m_{\nu}\right)_{e e}$ in terms of $C_{e e}^{(5)}$, the following restriction on $\Lambda$ is obtained

$$
\frac{\Lambda}{\left|C_{e e}^{(5)}\right|}>10^{11} \mathrm{TeV}
$$

The bound in (10) can be translated into a limit on the amplitude for $0 \nu \beta \beta$ decay at the 


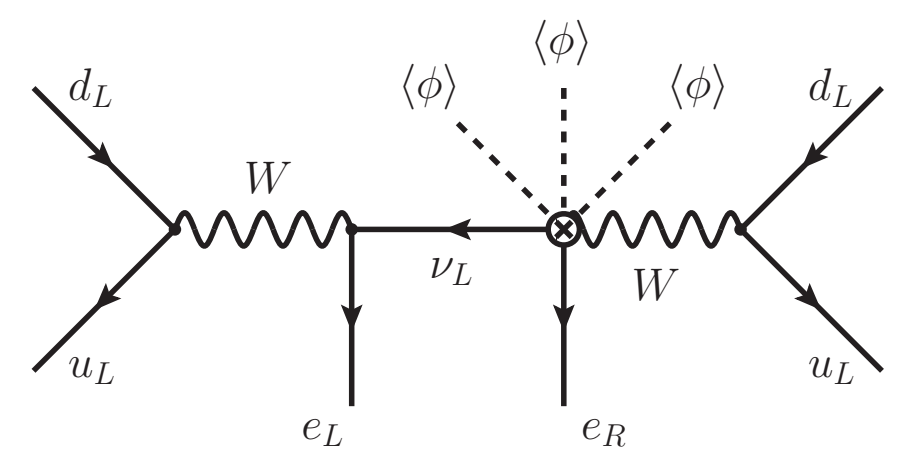

FIG. 3: Tree-level diagram contributing to $0 \nu \beta \beta$ decay with one $\mathcal{O}^{(7)}$ insertion.

parton level which can be estimated as

$$
\left|\mathcal{A}_{0 \nu \beta \beta}^{(5)}\right| \simeq \frac{G_{F}^{2}}{p_{\text {eff }}^{2}}\left|\left(m_{\nu}\right)_{e e}\right|,
$$

where $p_{\text {eff }} \sim 100 \mathrm{MeV}$ is the neutrino effective momentum obtained from averaging the corresponding nuclear matrix element contribution. Thus, from (10) one obtains

$$
\frac{p_{\text {eff }}}{G_{F}^{2}}\left|\mathcal{A}_{0 \nu \beta \beta}^{(5)}\right| \simeq \frac{\left|\left(m_{\nu}\right)_{e e}\right|}{p_{\text {eff }}}<5 \times 10^{-9},
$$

which is the limit on the $0 \nu \beta \beta$ decay amplitude that we will also impose in the other two cases.

\section{LNV operators with one LH lepton and one RH charged lepton}

The leading operator of this class, given in Eq. (3), has dimension 7 because it must involve two leptons, three scalar doublets to cancel the leptonic hypercharge, and one covariant derivative to compensate a chirality flip. Explicitly,

$$
\mathcal{O}_{e a}^{(7)}=\left(\phi^{\dagger} D^{\mu} \tilde{\phi}\right) \phi^{\dagger} \overline{e_{e R}} \gamma_{\mu} \tilde{\ell}_{a L} \rightarrow i \frac{g}{\sqrt{2}} v^{3} W_{\mu}^{-} \overline{e_{e R}} \gamma^{\mu} \nu_{a L}^{c}+\cdots .
$$

It must be noted, however, that in order to write simple, working models fulfilling all the experimental requirements, it may be necessary to impose additional symmetries, and consequently, some of the external (light) scalar doublet fields may not coincide with the SM Higgs doublet, as we mentioned above. This will be the case in the explicit model we will work out below.

The estimate from the $0 \nu \beta \beta$ decay amplitude shown in Fig. 3 is given by

$$
\left|\mathcal{A}_{0 \nu \beta \beta}^{(7)}\right| \simeq \frac{G_{F}^{2} v^{3}\left|C_{e e}^{(7)}\right|}{p_{\text {eff }} \Lambda^{3}} .
$$




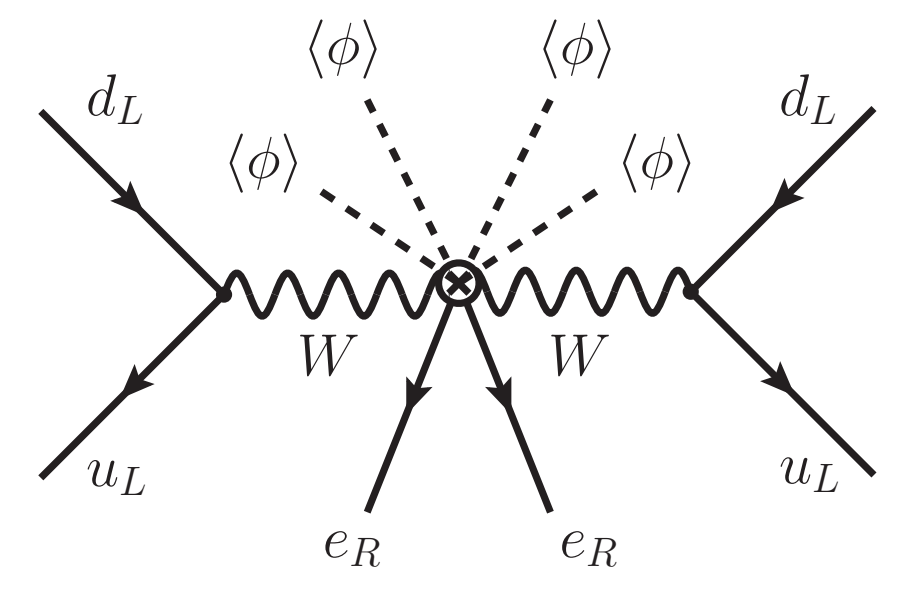

FIG. 4: Tree-level diagram contributing to $0 \nu \beta \beta$ decay with one $\mathcal{O}^{(9)}$ insertion.

This translates into a bound on $\Lambda$, which must be $>100\left|C_{e e}^{(7)}\right|^{1 / 3} \mathrm{TeV}$, if we want $\mathcal{A}_{0 \nu \beta \beta}^{(7)}$ to satisfy the limit in (13). These are order of magnitude estimates, but one can also use detailed nuclear matrix elements available in the literature [42, 43]. The interaction induced by the operator $\mathcal{O}^{(7)}$ can be partially expressed as a modification of the standard weak interaction, $W_{\mu} \bar{e} \gamma^{\mu}\left(\left(1-\gamma_{5}\right)+\eta\left(1+\gamma_{5}\right)\right) \nu$, where $\nu=\nu_{L}+\nu_{L}^{c}$ is a Majorana field. Then, the strong limit on $\eta$ derived using detailed nuclear matrix elements calculations, $|\eta|<4.4 \times 10^{-9}$ (see [42] and [43] where $\eta$ was termed $\epsilon_{V-A}^{V+A}$ ), reads in our case

$$
|\eta|=\frac{v^{3}}{\Lambda^{3}}\left|C_{e e}^{(7)}\right|<4.4 \times 10^{-9},
$$

implying a bound which is very close to our estimate

$$
\frac{\Lambda}{\left|C_{e e}^{(7)}\right|^{1 / 3}}>106 \mathrm{TeV} \text {. }
$$

\section{3. $L N V$ operators with two $R H$ charged leptons}

In this class the leading operator has dimension 9 and it is given in Eq. (4):

$$
\mathcal{O}_{e e}^{(9)}=\overline{e_{e R}} e_{e R}^{c}\left(\phi^{\dagger} D^{\mu} \tilde{\phi}\right)\left(\phi^{\dagger} D_{\mu} \tilde{\phi}\right) \rightarrow-\frac{g^{2}}{2} v^{4} W_{\mu}^{-} W^{-\mu} \overline{e_{e R}} e_{e R}^{c}+\cdots
$$

As we have argued in the companion paper [35], the $0 \nu \beta \beta$ decay amplitude in Fig. 4 can be large, near to its present experimental limit, with

$$
\left|\mathcal{A}_{0 \nu \beta \beta}^{(9)}\right| \simeq \frac{G_{F}^{2} v^{4} C_{e e}^{(9)}}{\Lambda^{5}} .
$$




\begin{tabular}{l|rcc} 
TeV & LL & LR & RR \\
\hline$\Lambda_{0 \nu \beta \beta}$ & $10^{11}$ & $10^{2}$ & 1
\end{tabular}

TABLE I: Natural (with $C_{e e}^{(n)} \sim 1$ ) NP scale limits for the three different lowest order effective operators mediating $0 \nu \beta \beta$ decay.

Requiring that the $\mathcal{A}_{0 \nu \beta \beta}^{(9)}$ amplitude satisfies the bound in Eq. (13), one obtains $\Lambda>$ $2\left|C_{e e}^{(9)}\right|^{1 / 5} \mathrm{TeV}$, but one can also make use of the detailed nuclear matrix element calculations. From $\mathcal{O}^{(9)}$ one obtains the following six-fermion contact interaction inducing $0 \nu \beta \beta$ decay

$$
\mathcal{L}_{0 \nu \beta \beta}=\frac{G_{F}^{2}}{2 m_{p}} \epsilon_{3}\left(\bar{u} \gamma^{\mu}\left(1-\gamma_{5}\right) d\right)\left(\bar{u} \gamma_{\mu}\left(1-\gamma_{5}\right) d\right) \bar{e}\left(1-\gamma_{5}\right) e^{c}
$$

where $m_{p}$ denotes the proton mass and in our case

$$
\epsilon_{3}=-\frac{2 m_{p} v^{4} C_{e e}^{(9)}}{\Lambda^{5}}
$$

This type of interaction has been studied in [44], where the bound $\left|\epsilon_{3}\right|<1.4 \times 10^{-8}$ at 90\% C.L. was found ${ }^{4}$. This also leads to a limit very close to our estimate

$$
\frac{\Lambda}{\left|C_{e e}^{(9)}\right|^{1 / 5}}>2.7 \mathrm{TeV}
$$

In Ref. [35] we present a realistic model which accommodates the observed neutrino masses and a large $0 \nu \beta \beta$ decay observable in the next round of experiments, proving explicitly the consistency of the previous estimates. In particular, neutrino masses are naturally suppressed even with NP at the TeV scale.

These scale estimates are summarized in the Table I. Note, however, that in actual models the operator coefficients $C_{e e}^{(n)}$ are not in general $\sim 1$ and the $\Lambda$ estimates may vary. For the LL case there are models with NP at $\Lambda \sim 1 \mathrm{TeV}$ for sufficiently suppressed couplings $C^{(5)} \sim 10^{-11}$ (see, for instance, Refs. [45-49]). We can also have different scales within the model, as in the LR model below, where the new leptons can have masses below a TeV and be observable at the LHC. Similarly, in the RR case in Ref. [35] the new scalar masses can range from few hundreds of $\mathrm{GeV}$ to tens of $\mathrm{TeV}$. A more detailed discussion can be found in the companion paper.

\footnotetext{
${ }^{4}$ There is a misprint in Ref. [44]. We thank the authors of this reference for providing us with the correct limit on $\epsilon_{3}$.
} 


\section{MAJORANA NEUTRINO MASSES GENERATED BY THE LNV OPERA- TORS INDUCING $0 \nu \beta \beta$ DECAY}

Once the effective theory generates one of the LNV operators that produce $0 \nu \beta \beta$ decay, neutrinos will get a mass at some (loop) order, even if there is no other independent source of neutrino masses. The three operators not only stand for NP at quite different mass scales but result in different neutrino mass structures. Specific models may of course include a combination of these effects, so that the final structure may be quite involved.

\section{LL operator}

After electroweak symmetry breaking $\mathcal{O}^{(5)}$ generates a well-known and much studied contribution to the neutrino masses (summation on repeated indices must be understood when applicable through the manuscript):

$$
\frac{C_{a b}^{(5)}}{\Lambda} \mathcal{O}_{a b}^{(5)}=\frac{C_{a b}^{(5)}}{\Lambda} \tilde{\ell}_{a L} \phi \tilde{\phi}^{\dagger} \ell_{b L} \rightarrow-\frac{v^{2} C_{a b}^{(5)}}{\Lambda} \overline{\nu_{a L}^{c}} \nu_{b L},
$$

where $a, b=e, \mu, \tau$ are flavor indices. Hence,

$$
\left(m_{\nu}\right)_{a b}=\frac{2 v^{2} C_{a b}^{(5)^{*}}}{\Lambda} .
$$

In this case $0 \nu \beta \beta$ decay proceeds through the diagram in Fig. 2, and it is proportional to

$$
\left|\left(m_{\nu}\right)_{e e}\right|=\left|U_{e i} m_{i} U_{i e}^{T}\right|=\left|c_{13}^{2} c_{12}^{2} e^{i \alpha_{1}} m_{1}+c_{13}^{2} s_{12}^{2} e^{i \alpha_{2}} m_{2}+s_{13}^{2} e^{-2 i \delta} m_{3}\right|
$$

where $U$ is the mixing matrix diagonalizing the neutrino mass matrix written in the current eigenstate basis with well-defined charged lepton flavor $[4,5]$ :

$$
m_{\nu}=\frac{2 v^{2} C^{(5)^{\dagger}}}{\Lambda}=U\left(\begin{array}{lll}
m_{1} & & \\
& m_{2} & \\
& & m_{3}
\end{array}\right) U^{T}
$$

and

$$
U=\left(\begin{array}{ccc}
c_{13} c_{12} & c_{13} s_{12} & s_{13} e^{-i \delta} \\
-c_{23} s_{12}-s_{23} s_{13} c_{12} e^{i \delta} & c_{23} c_{12}-s_{23} s_{13} s_{12} e^{i \delta} & s_{23} c_{13} \\
s_{23} s_{12}-c_{23} s_{13} c_{12} e^{i \delta} & -s_{23} c_{12}-c_{23} s_{13} s_{12} e^{i \delta} & c_{23} c_{13}
\end{array}\right)\left(\begin{array}{ll}
e^{i \alpha_{1} / 2} & \\
& e^{i \alpha_{2} / 2} \\
& 1
\end{array}\right)
$$


where $s_{i j} \equiv \sin \theta_{i j}$ and $c_{i j} \equiv \cos \theta_{i j}$. Thus $\Lambda$ is required to be $\sim 6 \times 10^{11} \mathrm{TeV}$ to reproduce the observed neutrino masses $\left(m_{\nu}\right)_{\tau \tau} \sim 0.1 \mathrm{eV}$ for $C_{a b}^{(5)} \sim 1$, which is of the same order as the limit derived from $0 \nu \beta \beta$ decay (see Table I).

\section{LR operator}

Restoring flavor indices the LR contribution to the effective Lagrangian becomes

$$
\frac{C_{a b}^{(7)}}{\Lambda^{3}}\left(\phi^{\dagger} D^{\mu} \tilde{\phi}\right)\left(\phi^{\dagger} \overline{e_{a R}} \gamma_{\mu} \tilde{\ell}_{b L}\right)
$$

In this case the neutrino masses are generated by radiative corrections (left graph in Fig. 1). Here it is important to differentiate between the calculable (logarithmic) contributions to the masses derived from $\mathcal{O}^{(7)}$ and the estimates obtained by matching. The first, obtained in standard effective field-theory fashion using dimensional regularization and a renormalizable gauge, scale like

$$
\left(\delta m_{\nu}\right)_{a b} \simeq \frac{v^{3}}{16 \pi^{2} \Lambda^{3}}\left(m_{a} C_{a b}^{(7)}+m_{b} C_{b a}^{(7)}\right) \log \left(\frac{\Lambda}{v}\right) .
$$

Whereas the estimates from matching, which give the dominant contribution to neutrino masses, are obtained using dimensional analysis and are of the form

$$
\left(m_{\nu}\right)_{a b} \sim \frac{v}{16 \pi^{2} \Lambda}\left(m_{a} C_{a b}^{(7)}+m_{b} C_{b a}^{(7)}\right),
$$

as we will derive for the specific models discussed below. Note, however, that even for this last estimate it is important to use a renormalizable gauge as in the unitary gauge spurious positive powers of $\Lambda$ may appear. That the final result must be proportional to $1 / \Lambda$ follows from (i) the fact that the dimension 5 Weinberg operator is the only one describing neutrino Majorana masses (in the absence of $\nu_{R}$ ); and (ii) that we assume that the NP is decoupling. It is also important to note that, as mentioned in the introduction, specific models may have more than one NP scale so that $\Lambda$ represents an effective scale and may not correspond to the mass of any specific particle. For example, if the theory has two scales $M>M^{\prime}$ one may have $\Lambda=M^{2} / M^{\prime}$; we will present an example of this situation in Section V 2 .

The expression (30) coincides with the results obtained in specific models such as the one worked-out in Section V 2 (Eq. (41)) with the appropriate identification of $\Lambda$. In this case $\left(m_{\nu}\right)_{\tau \tau} \sim 0.1 \mathrm{eV}$ with $C_{\tau \tau}^{(7)} \sim 1$ implies (see Eq. (6)) $\Lambda \sim 4 \times 10^{7} \mathrm{TeV}$, that should be compared to the limit obtained from $0 \nu \beta \beta$ decay in Table I, which is several orders of 
magnitude smaller. This implies that in realistic models the coefficients $C^{(7)}$ must be much less than 1 to allow for a $\Lambda$ of the order of $100 \mathrm{TeV}$, in agreement with the $0 \nu \beta \beta$ decay estimate, if this is to be observed in the next generation of experiments. This is what happens in the explicit model we will work out below.

\section{3. $R R$ operator}

Finally, the LNV operator $\mathcal{O}^{(9)}$ generates Majorana masses to neutrinos at two loops, in this case also suppressed but by two loop factors and two charged lepton mass insertions (see the right panel of Fig. 1). As for the LR case we distinguish between the calculable (logarithmic) contributions,

$$
\left(\delta m_{\nu}\right)_{a b} \simeq \frac{v^{4}}{\left(16 \pi^{2}\right)^{2} \Lambda^{5}} m_{a} C_{a b}^{(9)} m_{b} \log \left(\frac{\Lambda}{v}\right)
$$

and the estimates obtained from matching

$$
\left(m_{\nu}\right)_{a b} \sim \frac{1}{\left(16 \pi^{2}\right)^{2} \Lambda} m_{a} C_{a b}^{(9)} m_{b} .
$$

Explicit calculations in specific models (using renormalizable gauges) [35] reproduce (32) up to a proportionality factor of order 1 that depends on the various masses in the loop, and when $\Lambda$ is identified with an appropriate combination of heavy scales. For $\left(m_{\nu}\right)_{\tau \tau} \sim 0.1 \mathrm{eV}$ and $C_{\tau \tau}^{(9)} \sim 1,(32)$ implies $\Lambda \sim 1.3 \times 10^{3} \mathrm{TeV}$, several orders of magnitude larger than the $0 \nu \beta \beta$ decay estimate in Table I. Although in realistic models the coefficients $C^{(9)}$ and $\Lambda$ are typically smaller [35].

\section{HEAVY PARTICLE ADDITIONS GENERATING THE LOWEST ORDER LNV OPERATORS AT TREE LEVEL}

In this section we will work out the combinations of heavy particles (scalars, fermions and gauge bosons) that must be present in any extension of the SM if it is to generate one of the operators $\mathcal{O}^{(5,7,9)}$ at tree level; thus producing the largest possible rates for $0 \nu \beta \beta$ decay. We will assume that the underlying theory is weakly coupled and contains only renormalizable vertices ${ }^{5}$; we also assume the NP respects all the gauge symmetries of the SM. In listing the

\footnotetext{
${ }^{5}$ Non-renormalizable vertices are presumably suppressed by inverse powers of a yet higher scale $\Lambda_{\text {high }}$ that
} we assume much larger than $\Lambda$. 

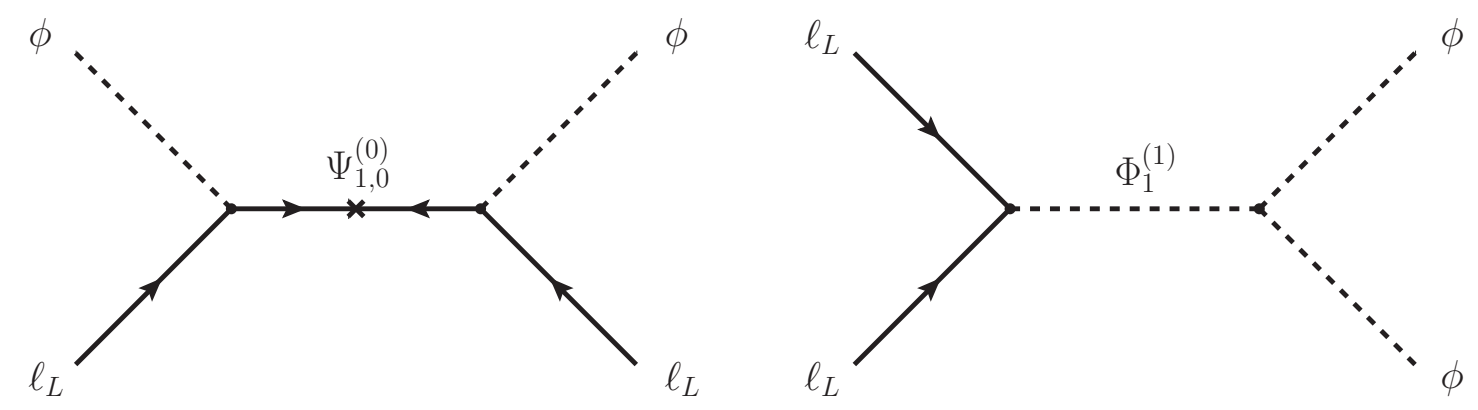

FIG. 5: Topologies generating $\mathcal{O}^{(5)}$. These graphs together with those with one or two $W$ boson attachments generate $\mathcal{O}^{(7-\mathrm{I}, 7-\mathrm{II}, 7-\mathrm{III})}$.

heavy particles we denote by $X_{I}^{(Y)}, \Psi_{I}^{(Y)}$ and $\Phi_{I}^{(Y)}$ a heavy vector, fermion or scalar with isospin $I$ and hypercharge $Y$, respectively. When the heavy particles can be either a heavy vector or heavy scalar with the same isospin and hypercharge, we use $B_{I}^{(Y)}$ to denote both possibilities.

\section{LL additions}

In the case of $L N V$ operators with two LH leptons the heavy excitations that can generate the operators of dimension 5 and 7 in Eqs. (8) and (9), respectively, are

$$
\Phi_{1}^{(1)} ; \quad \Psi_{1,0}^{(0)}
$$

(If the model has two or more light scalar isodoublets, a heavy scalar singlet $\Phi_{0}^{(1)}$ can also generate these operators [50].) This means that, as mentioned previously, if the underlying theory generates (9), it will also generate (8) with the same heavy scale. In Fig. 5 we depict the diagram topologies resulting in those operators upon integration of the heavy particles flowing through the internal lines.

\section{LR additions}

The sets of heavy excitations that can generate the dimension $7 \mathrm{LNV}$ operator with one LH lepton and one RH electron in Eq. (14) are

$$
\left\{X_{1 / 2}^{(3 / 2)}, B_{0,1}^{(1)}\right\} ; \quad\left\{\Psi_{1 / 2}^{(1 / 2)}, B_{0,1}^{(1)}\right\} ; \quad\left\{\Psi_{1 / 2}^{(1 / 2)}, \Psi_{0,1}^{(0)}\right\} ; \quad\left\{\Psi_{0}^{(0)}, B_{0}^{(1)}\right\} ; \quad\left\{\Psi_{1}^{(0)}, B_{1}^{(1)}\right\}
$$


In this case one must always exchange two heavy particles. It is worth noting that in the last two possibilities the heavy fermions $\Psi_{0,1}^{(0)}$ necessarily have couplings that would also generate the operators in Eqs. (8) and (9) at tree level. In contrast, models containing $\Phi_{0,1}^{(1)}$ may or may not generate them, depending on whether the heavy scalars couple to $\ell_{L} \times \ell_{L}$ (which may be forbidden by the symmetries of the underlying theory). In the appendix we provide the diagram topologies relevant to each case.

One can construct many models choosing from the above NP matter contents. However, in order to make the model phenomenologically viable we must often enlarge these minimal sets. This is because (34) are fixed only by the requirement that they generate $\mathcal{O}^{(7)}$ at tree level, which does not insure the preservation of extra symmetries that are sometimes necessary, for instance, to avoid too large LFV rates, or to forbid tree-level neutrino masses, or to reproduce the observed lepton spectrum. In Section V we discuss a realistic, simple example for the case $\left\{\Psi_{1 / 2}^{(1 / 2)}, \Phi_{1}^{(1)}\right\}$. But, as we will argue, for the model to be realistic, it must include at least two fermion doublets $\Psi_{1 / 2}^{(1 / 2)}$ besides one scalar triplet $\Phi_{1}^{(1)}$, and, in addition, a second light scalar doublet $\phi^{\prime}$.

\section{RR additions}

Finally, the sets of heavy excitations that can generate at tree level the dimension $9 \mathrm{LNV}$ operator with two RH electrons in Eq. (18) are

$$
\begin{aligned}
& \left\{\Phi_{0}^{(2)}, B_{1 / 2}^{(3 / 2)}, B_{0,1}^{(1)}\right\} ; \quad\left\{\Phi_{0}^{(2)}, B_{0,1}^{(1)}\right\} ; \quad\left\{\Psi_{1 / 2}^{(1 / 2)}, B_{1 / 2}^{(3 / 2)}, B_{0,1}^{(1)}\right\} ; \\
& \left\{\Psi_{1 / 2}^{(1 / 2)}, \Psi_{1}^{(0)}, B_{0,1}^{(1)}\right\} ; \quad\left\{\Psi_{1 / 2}^{(1 / 2)}, B_{0,1}^{(1)}\right\} ; \quad\left\{\Psi_{1}^{(0)}, B_{1}^{(1)}\right\} ; \quad\left\{\Psi_{0}^{(0)}, B_{0}^{(1)}\right\} .
\end{aligned}
$$

Despite the presence of $\Phi_{0,1}^{(1)}$ and $\Psi_{0,1}^{(0)}$ in some of these options, these heavy particles need not have the same vertices as the ones leading to (8). If they do, $\mathcal{O}^{(9)}$ would have only subdominant effects; but this is in general not the case. In the appendix we also provide the diagram topologies relevant to each of these cases.

There are many models that can be constructed containing the above particle content. For example, the case where the scalar sector of the SM is extended by adding a doublycharged isosinglet $\Phi_{0}^{(2)}$ and an isotriplet of unit hypercharge $\Phi_{1}^{(1)}$, was considered in detail in the companion paper [35]. (See also $[51,52]$.) As in other cases, additional heavy fields may be required in order to make the model realistic. 


\section{SIMPLE EXAMPLES OF FUNDAMENTAL THEORIES WITH A LARGE $0 \nu \beta \beta$ DECAY RATE AND REALISTIC NEUTRINO MASSES}

There are many specific models fulfilling the three generic scenarios discussed above (see Eqs. (2),(3) and (4)). Here we list a few for illustration purposes; we do not aim at reviewing all models that have been considered in the literature; concentrating instead on specific examples that exhibit the salient features discussed previously. In particular neutrino masses take the form in Eqs. (5), (6) and (7), and in general the coefficients $C^{(n)} \ll 1$ for consistency in realistic models.

\section{LL models}

SM extensions that at tree level generate the Weinberg operator $\mathcal{O}^{(5)}$ at low energies, have been extensively studied in the literature (for type I see-saw see [27-30], for see-saw type II see [53-58], and for see-saw type III see [59, 60]). Specific models for any of the three possible see-saw scenarios ${ }^{6}$ (see Eq. (33)) require very heavy mediators, with masses $\sim 10^{14} \mathrm{GeV}$ or very small couplings, and were discussed some time ago (for recent reviews see $[2,3,61,62])$. The most popular and simplest case, and also the pioneering one, results from the addition of heavy $\mathrm{RH}$ neutrinos $\Psi_{0}^{(0)}=\nu_{R}$ with the renormalizable Lagrangian

$$
\mathcal{L}^{\nu_{R}}=i \overline{\nu_{a R}} \not \nu_{a R}-\left\{\frac{1}{2} M_{a} \overline{\nu_{a R}^{c}} \nu_{a R}-y_{a b} \overline{\nu_{a R}} \tilde{\phi}^{\dagger} \ell_{b L}+\text { h.c. }\right\}
$$

including the kinetic terms and the Yukawa couplings $y_{a b}$. There must be at least two heavy neutrinos $\nu_{a R}$ (with masses $M_{a}$ ) to guarantee that the light neutrino mass matrix is at least of rank 2, required in order to account for the three non-degenerate light neutrinos. Explicitly,

$$
\left(m_{\nu}\right)_{a b}=\frac{2 v^{2} C_{a b}^{(5) *}}{\Lambda}=-\frac{y_{c a}^{*} y_{c b}^{*}}{M_{c}} v^{2}, \text { with }\left|\frac{C_{a b}^{(5)}}{\Lambda}\right| \lesssim 10^{-11} \mathrm{TeV}^{-1},
$$

in agreement with Eq. (11) and Table I. The alternative case with the see-saw messengers near the TeV scale (and $|y|<10^{-5}$ ) has become more popular with the launch of the LHC, see $[48,63]$, and references therein.

In this scenario $0 \nu \beta \beta$ decay follows from the exchange of the light Majorana neutrinos; the resulting amplitude is proportional to the effective electron-neutrino mass $\left(m_{\nu}\right)_{e e}$ in Eq. (25),

\footnotetext{
${ }^{6}$ Assuming only one light SM Higgs doublet.
} 
see Fig. 2; hence, any $0 \nu \beta \beta$ decay rate within the present experimental precision can be accommodated. Indeed, a global fit to neutrino oscillation data gives (see, for instance, [6]) $\Delta m_{21}^{2} \equiv m_{2}^{2}-m_{1}^{2}=\left(7.59_{-0.18}^{+0.20}\right) \times 10^{-5} \mathrm{eV}^{2}, \Delta m_{31}^{2} \equiv m_{3}^{2}-m_{1}^{2}=\left(2.50_{-0.16}^{+0.09}\right) \times 10^{-3} \mathrm{eV}^{2}$, $s_{12}^{2}=0.312_{-0.015}^{+0.017}, s_{23}^{2}=0.52_{-0.07}^{+0.06}, s_{13}^{2}=0.013_{-0.005}^{+0.007}$. Replacing these values into Eq. (25) one can obtain at $1 \sigma$ any $0 \nu \beta \beta$ decay rate compatible with present experimental limits for the normal hierarchy, although it is bounded from below for the inverse one $[1,64,65]$.

\section{LR models}

To our knowledge, no realization of this second scenario has been spelled out in the literature. One can construct many models with $0 \nu \beta \beta$ decay into two electrons of opposite chirality mediated by $\mathcal{O}^{(7)}$ by choosing among the matter contents in Eq. (34); however, when constructing a realistic model we must in general enlarge these minimal sets.

We start from the set $\left\{\Psi_{1 / 2}^{(1 / 2)}, \Phi_{1}^{(1)}\right\}$ contained in Eq. (34); to simplify the notation we

define $\Phi_{1}^{(1)} \equiv \chi$, a scalar isotriplet of hypercharge 1 , and $\Psi_{1 / 2}^{(1 / 2)} \equiv L^{c}=L_{L}^{c}+L_{R}^{c}$, a lepton isodoublet of hypercharge 1/2 (in terms of its LH and RH components); a simple way to insure the decoupling of the heavy physics is to assume, as we do, that the heavy fermions are vector like. This particle content is sufficient to generate $\mathcal{O}^{(7)}$ at tree level, and it is not hard to convince oneself (see the appendix) that the relevant graphs must involve the couplings $e_{R} \phi \tilde{L}, \ell_{L} L \chi$ and $\phi^{\dagger} \phi^{\dagger} \chi$. However, such a model also allows the coupling $\ell_{L} \ell_{L} \chi$ and will then generate $\mathcal{O}^{(5)}$ at tree level through the standard type-II see-saw diagram (on the right of Fig. 5), a possibility we wish to disallow. In order to do this we impose a discrete $Z_{2}$ symmetry under which $\chi$ and $L$ are odd and $\ell_{L}$ is even, so $\mathcal{O}^{(5)}$ does not appear at tree level; unfortunately, this symmetry also forbids the $\phi^{\dagger} \phi^{\dagger} \chi$ vertex . In order to overcome this difficulty we assume the presence of two light scalar doublets $\phi, \phi^{\prime}$ which are, respectively, even and odd under $Z_{2}$. The allowed vertices are then

$$
\overline{\tilde{L}} \chi \ell_{L}, \quad \bar{L} \phi^{\prime} e_{R}, \quad \phi^{\dagger} \chi \tilde{\phi}^{\prime}
$$

from which $\mathcal{O}^{(7)}$ is generated through diagrams such as the one in Fig. 6, while $\mathcal{O}^{(5)}$ appears only at one loop.

As we will explain below, in order to accommodate a generic neutrino mass matrix and also allow for flavor symmetries treating the three families on the same footing, we will 


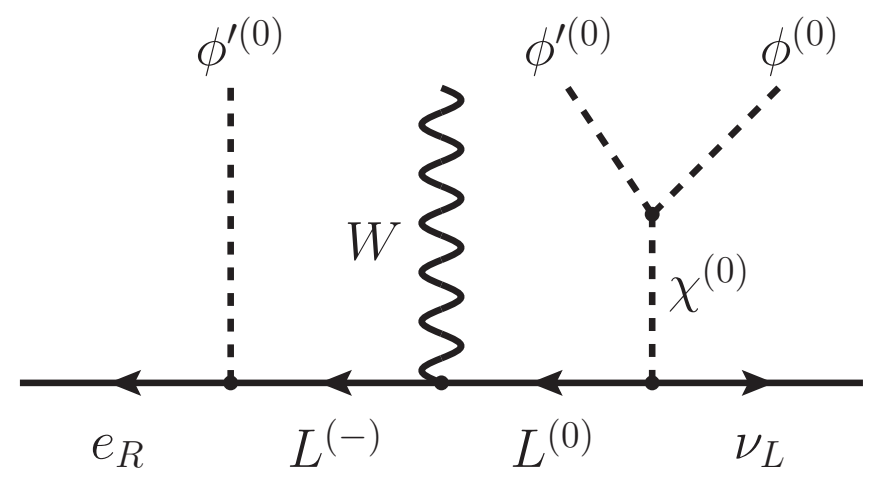

FIG. 6: Tree-level diagram contributing to $\mathcal{O}^{(7)}$ in the model proposed.

\begin{tabular}{l|cccc} 
& $L_{L a}$ & $L_{R a}$ & $\chi$ & $\phi^{\prime}$ \\
\hline$S U(2)_{L}$ & $\frac{1}{2}$ & $\frac{1}{2}$ & 1 & $\frac{1}{2}$ \\
$U(1)_{Y}$ & $-\frac{1}{2}$ & $-\frac{1}{2}$ & 1 & $\frac{1}{2}$ \\
$Z_{2}$ & - & - & - & -
\end{tabular}

TABLE II: Quantum number assignment for the extra fields.

assume the presence of 3 heavy vector-like fermion doublets $L_{a}, a=1,2,3$. The complete list of new fields is given in Table II; the Lagrangian will include renormalizable couplings preserving these symmetries, noting that the SM fields transform trivially under $Z_{2}$. The second scalar doublet $\phi^{\prime}$ could be identified with the isodoublet giving mass to the up quark sector in two-doublet models [66-71] (for reviews see for instance [72, 73]) if we require the RH up-quark singlets to be odd under $Z_{2}$ (the quark isodoublets are even). We will prefer not to do so, because we will find it convenient to be able to assign a small VEV $\left\langle\phi^{\prime}\right\rangle$. We also note that in this model LN is explicitly broken by (renormalizable) terms in the scalar potential, in particular by $\left(\phi^{\dagger} \phi^{\prime}\right)^{2}$.

The scalar potential can be easily arranged to insure a minimum where $\langle\phi\rangle \gg\left\langle\phi^{\prime}\right\rangle,\langle\chi\rangle \neq$ 0 , with $\langle\chi\rangle \simeq-\mu^{*}\left\langle\phi^{\prime}\right\rangle\langle\phi\rangle / m_{\chi}^{2}, \mu$ the trilinear $\phi^{\dagger} \chi \tilde{\phi}^{\prime}$ coupling and $m_{\chi}$ the isotriplet mass (in order to satisfy the limit from electroweak precision data $[1,74]$ we require $\langle\chi\rangle \lesssim 2$ $\mathrm{GeV}$; see [35] for a similar analysis and [75] for a recent study, in the framework of the type II see-saw, including one-loop radiative corrections from the scalars of the model). We assume negative mass terms for $\phi$ and $\phi^{\prime}$ to trigger the corresponding VEVs, whereas $\chi$ gets a VEV through its mixing with the scalar isodoublets. Otherwise, dimensional couplings 
in the potential are typically of electroweak order, except for new scalar masses that may be larger. Dimensionless ones stay perturbative, in general ranging within an $\alpha_{E M} \sim 10^{-2}$ factor.

We now discuss briefly the viability of the model, concentrating on the effects of the masses and mixings of the new fermions, the induced LFV effects, and the implications for both the LHC, and the light neutrino masses. The heavy lepton Lagrangian reads

$$
\mathcal{L}_{\mathrm{H}}^{L}=\overline{L_{a}}\left(i \not \varnothing-M_{a}\right) L_{a}+\left\{y_{a b}^{e} \overline{L_{a L}} \phi^{\prime} e_{b R}+y_{a b}^{\nu} \overline{\tilde{L}_{a L}} \chi \ell_{b L}+\text { h.c. }\right\}
$$

where we assumed that the heavy mass matrix is diagonal without loss of generality. Once $\phi^{\prime}$ and $\chi$ acquire VEVs the light $e_{R}, \ell_{L}$ leptons mix with the $L_{a}$; such mixings and the corresponding phenomenology of heavy vector-like lepton doublets were analysed long ago [76-78], and more recently within the context of Little Higgs models [79-82], and Extra Dimensional theories [83-85] (for a review see [86, 87]; for updated limits see [88]).

The low-energy effects of such mixings will be proportional to $y_{a b}^{e}\left\langle\phi^{\prime}\right\rangle / M_{a}$ or $y_{a b}^{\nu}\langle\chi\rangle / M_{a}$ and can be made as small as experimentally required by increasing the heavy masses $M_{a}$, reducing the couplings $y_{a b}^{e, \nu}$, or the $\operatorname{VEVs}\left\langle\phi^{\prime}\right\rangle,\langle\chi\rangle$. LFV effects can be further suppressed by assuming that the light charged leptons, which get their masses through the SM Higgs mechanism, are aligned along the heavy flavors. This corresponds to taking $y_{a b}^{e}$ diagonal, which may be natural in a larger model. The LHC reach for the scalar triplet was reviewed in the companion paper and is updated in next section. The production of the $L_{a}$ at the LHC has been also studied previously [89] and the general conclusion is that they will be detected provided their masses are below $850 \mathrm{GeV}$ for a center of mass (CM) energy of $14 \mathrm{TeV}$ and an integrated luminosity of $100 \mathrm{fb}^{-1}$ [90] (the LHC reach reduces to $350 \mathrm{GeV}$ for heavy leptons mainly decaying into taus [91]).

Given the couplings of the model one can evaluate $C_{a b}^{(7)}$ by using the diagram in Fig. 6,

$$
\frac{C_{a b}^{(7)}}{\Lambda^{3}}=-i \frac{\mu y_{c a}^{e *} y_{c b}^{\nu *}}{m_{\chi}^{2} M_{c}^{2}}
$$

where all masses in the $L_{c}$ and $\chi$ multiplets are taken equal.

Light neutrinos are massless at tree level, but they get a mass at one loop through diagrams like that in Fig. 7. The full calculation gives

$$
\left(m_{\nu}\right)_{a b} \simeq \frac{v^{\prime 2} \mu}{32 \pi^{2} v}\left(m_{a} y_{c a}^{e *} y_{c b}^{\nu *}+m_{b} y_{c b}^{e *} y_{c a}^{\nu *}\right) \frac{1}{M_{c}^{2}-m_{\chi}^{2}} \log \frac{M_{c}^{2}}{m_{\chi}^{2}}
$$




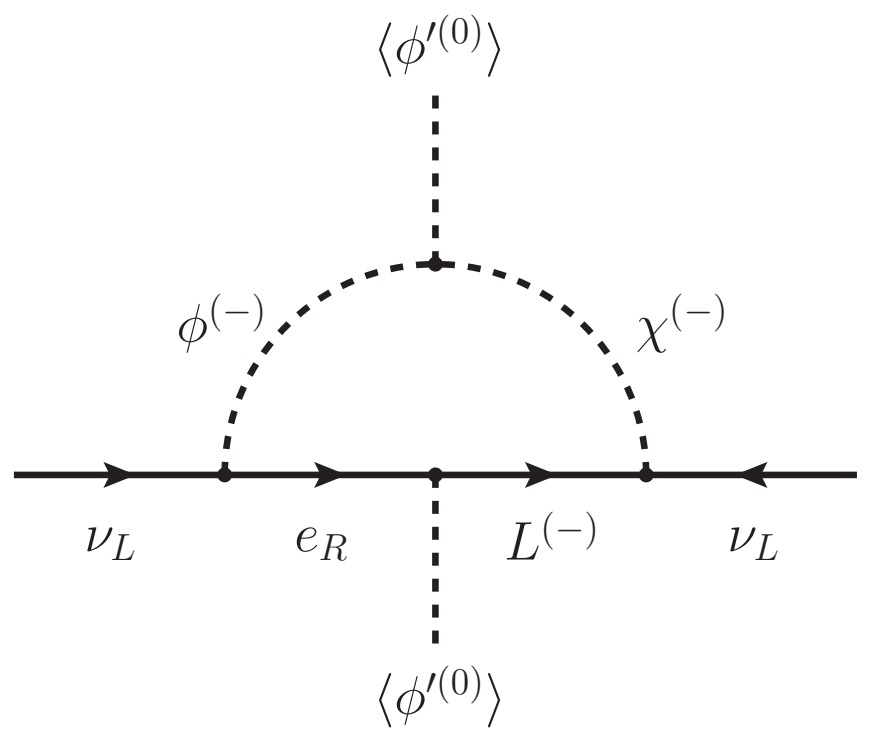

FIG. 7: Leading one-loop contribution in the Feynman gauge to neutrino masses in the fundamental theory.

where $v^{\prime}=\left\langle\phi^{\prime}\right\rangle$ and we have assumed that all other masses are much smaller than $M_{c}$ and $m_{\chi}$. Thus, with only one heavy lepton doublet the neutrino mass matrix has at most rank 2 . With two heavy lepton doublets all three light neutrinos can be massive, but with three it is also straightforward to impose a flavor symmetry that forbids any potentially large LFV.

The model may face a domain wall problem if the spontaneously broken $Z_{2}$ symmetry is exact. This can be obviated adding a softly breaking term $\phi^{\dagger} \phi^{\prime}$ to the scalar potential. In a future publication we will provide a detailed analysis of this model including the neutrino mass calculation, as well as the quantitative discussion of the parameter space allowed by present experimental bounds, and the predictions for the different observables.

\section{RR models}

The final case, where $0 \nu \beta \beta$ decay involves two $\mathrm{RH}$ electrons through the operator $\mathcal{O}^{(9)}$, allows for many tree-level realizations, typically with a heavy sector near the electroweak scale as argued in Section IV and in the appendix. A realistic, simple model of this scenario with the neutrino masses generated at two loops, and predicting a non-zero third mixing angle $\sin ^{2} \theta_{13} \gtrsim 0.008$ for a large $0 \nu \beta \beta$ decay rate, was studied thoroughly in the companion paper [35] and corresponds to the particular SM addition $\left\{\Phi_{0}^{(2)}, \Phi_{1}^{(1)}\right\}$ in Eq. (35). The model 
contains in addition a real scalar singlet $\sigma$ whose presence allows a $Z_{2}$ discrete symmetry that protects the neutrinos from acquiring a tree-level mass. This, in turn, makes the neutrino masses calculable.

\section{OTHER PHENOMENOLOGICAL IMPLICATIONS}

Although it is beyond the scope of this paper to make a detailed discussion of the experimental constraints on the different scenarios and models, we will briefly comment on the main phenomenological implications for ongoing and forthcoming experiments. The present study answers the general question of how large can the $0 \nu \beta \beta$ amplitude be, requiring that the only light fields are those of the SM, and assuming that the NP responsible for the

effect is weakly coupled and has a characteristic scale above the electroweak scale. In particular this means then that neutrino masses are Majorana; we also assume, as it is widely believed, that the observed pattern of neutrino oscillations indicates the presence of three massive neutrinos [1]. This strongly influences our analysis because we must then explain the neutrino spectrum, assuming that there is no other larger source of LNV beyond the one mediating $0 \nu \beta \beta$ decay.

Thus, within this framework, we have to verify on a case by case basis whether:

- The $0 \nu \beta \beta$ decay rate can be fast enough to be observable at the next round of experiments.

- The neutrino masses are correctly predicted.

- LFV and universality limits are within experimental bounds.

- The new heavy particles satisfy the collider exclusion limits.

We have already addressed the first two points in general and in some detail; we consider the remaining two in the following subsections.

\section{A. LFV processes in SM extensions with a sizeable $0 \nu \beta \beta$ decay rate}

A relatively large $0 \nu \beta \beta$ decay rate requires a not too heavy NP (except for the case where $\mathcal{O}^{(5)}$ is generated at tree level). This, in turn, implies that the effects of the new 
particles may be detected in highly suppressed processes, like in flavor-changing leptonic or $Z$ decays [1]; the corresponding constraints are model dependent, however. We can compare, for illustration, the LR and RR models in the previous section and in Ref. [35], respectively. In the latter the heavy sector only involves new scalars, with little effect on lepton universality precision tests, for instance. The main universal constraint derived from them being the upper bound on the isotriplet $\mathrm{VEV},\langle\chi\rangle \lesssim 2 \mathrm{GeV}[1,74]$. This limit is easy to satisfy although it is rather restrictive in this specific model due to the small number of new free parameters available, and the desirability of accommodating a large $0 \nu \beta \beta$ decay rate. For the same reason LFV constraints are quite demanding because the corresponding leptonic decays are proportional to neutrino masses, which have a large misalignment from the charged lepton current eigenstates, described by the Pontecorvo-Maki-Nakagawa-Sakata mixing matrix $[4,5]$. This translates into lower bounds on the heavy scalar masses with a preference for values above a $\mathrm{TeV}$ [35].

The LR model presented in this manuscript is somewhat different, for the new scalars have no direct coupling to SM lepton pairs, although they are induced by fermion mixing. In this case the most stringent restrictions concern the new heavy leptons, with masses $M_{a}$, especially on their mixing with the light ones; such indirect constraints on extra vector-like leptons have been thoroughly studied in the literature [76-88]. The vector-like character that allows their decoupling without breaking the SM, also fixes their mixing behavior and their low energy phenomenology. The corrections to SM vertices are suppressed by at least 2 powers of a small ratio $\mathcal{O}\left(m_{\ell} / M_{a}\right)$, where $m_{\ell}$ is typically a light lepton mass. Hence, the decay rates, which are vanishingly small within the SM, are suppressed by at least 4 powers of these ratios; moreover, they also vanish when $\left\langle\phi^{\prime}\right\rangle \rightarrow 0$. Even more, LFV processes can be also canceled by aligning the heavy and light lepton flavors. In summary, many different small factors can conspire to make negligible the effects of the heavy fermion, in particular, their tree-level effects are naturally small enough to accommodate the experimental constraints. The main restrictions on this type of models result from one-loop contributions exchanging heavy leptons and bosons; the most restrictive processes being those including the muon to electron transition. In fact, one can, to a large extent, apply the conclusions from related analyses for the Littlest Higgs model with T-parity [79-82]; the general conclusion is that the heavy flavors must be aligned with the light charged leptons with a precision better than $1-10 \%$ for heavy masses of $\mathcal{O}(\mathrm{TeV})$. 
In summary, LFV provide stringent restrictions on these models but they can be satisfied within relatively large regions of parameter space. What would be more interesting, these models could also explain a departure from the SM predictions if found in the ongoing and forthcoming searches for LFV [1, 92] (see [93] for a review).

\section{B. Collider searches for particles with LNV interactions}

The specific models above also have different collider signatures: the LR model contains heavy fermions and scalars, while the RR model contains only heavy scalars; all of which can be searched for at the LHC. Verification of either model would involve not only the discovery of the corresponding heavy particles, but also a demonstration of the presence of LNV interactions. (the possibility of observing LNV events at LHC was emphasized quite some time ago [94]). Although not all decays of the new particles produce LNV signals, in some cases they may be dominant; however, in general one has to search for the new resonances in the most sensitive channels, and only afterwards address the possibility of observing LNV events. Typically, these will be difficult to observe because LNV is usually a small effect since the corresponding amplitudes involve several (small) couplings all of which must be present in order for $\mathrm{LN}$ to be broken. Hence, in general the dominant production mechanisms are standard and LN conserving, otherwise they are small (though LNV decays can be slow, they typically still can occur within the detector [95]).

Following the discussion in Ref. [35] we will first comment on the detection of the new scalars in the RR model. Doubly-charged scalars have fixed couplings to photons, so that their production cross section is known; their decay into leptons (if allowed) gives a very clean signal, which is particularly important at hadronic machines; although it is not LNV by itself. Therefore, if doubly-charged scalars are light enough, they are quite suited for detection at colliders. Generally, this type of scalars forms part of a weak triplet, and usually also acts as see-saw messenger of type II $[53-58,96]$ (see also [97]). These triplets are then theoretically well-motivated, especially when considering L-R symmetric models, and simulations of their production at future colliders can be found in the literature [98-102] (see also [103, 104] for recent studies; and for model-independent ones $[105,106])$. The general conclusion is that the LHC discovery limit can reach masses over $600 \mathrm{GeV}$ (for a CM energy of $14 \mathrm{TeV}$ and an integrated luminosity of $30 \mathrm{fb}^{-1}$ ) [103, 107]; although the actual limits may be larger 
given the outstanding LHC performance for a CM energy of $7 \mathrm{TeV}$ [108]. (See for a recent review [109].) First results from CMS [110] and ATLAS [111] have been presented at this last CM energy with an integrated luminosity of $0.89 \mathrm{fb}^{-1}$ for any di-lepton scalar final state and of $1.6 \mathrm{fb}^{-1}$ for di-muon final states, respectively. As nothing is seen, a lower bound on the doubly-charged scalar mass is obtained: of about $250 \mathrm{GeV}$ if the main decay channels contain $\tau$ leptons and to about $300 \mathrm{GeV}$ if they contain only electrons or muons [110], reaching $375 \mathrm{GeV}$ if they only couple to muons [111]. (Present Fermilab Tevatron Collider limits are less stringent $[112,113]$.) In our case, however, the doubly-charged triplet, $\chi^{ \pm \pm}$ does not directly couple to fermions; while the other doubly-charged scalar in the model, a singlet $\kappa^{ \pm \pm}$, does not couple to $W$ pairs. However, they mix; and both of them can be produced at LHC via the Drell-Yan mechanism $\left(q \bar{q} \rightarrow \gamma^{*}, Z^{*} \rightarrow \chi^{++} \chi^{--}, \kappa^{++} \kappa^{--}\right)$. Since this is the main production process assumed by both LHC Collaborations, the former limits apply directly to the singlet decaying dominantly to lepton pairs for a small mixing: $m_{\kappa}>$ $300 \mathrm{GeV}$. Limits on the triplet mass will be more difficult to derive, for the dominant process $q \bar{q} \rightarrow \gamma^{*}, Z^{*} \rightarrow \chi^{++} \chi^{--} \rightarrow W^{+} W^{+} W^{-} W^{-}$is more complicated to study, due to its large backgrounds and the inherent difficulty of reconstructing several leptonic $W$ decays [114]. The only viable LNV decay channels $q \bar{q} \rightarrow \gamma^{*}, Z^{*} \rightarrow \chi^{++} \chi^{--}, \kappa^{++} \kappa^{--} \rightarrow e_{a}^{ \pm} e_{b}^{ \pm} W^{\mp} W^{\mp}$ are suppressed by the small mixing between $\chi^{ \pm \pm}$and $\kappa^{ \pm \pm}$.

The LR model discussed above contains, besides the scalar isotriplet of unit hypercharge $\chi$, a second scalar isodoublet and several vector-like lepton doublets. This scalar triplet couples to a light and a heavy lepton, and the latter decays into a light lepton and a $W$, or a $Z$, or a Higgs boson; this results in a four-fermion decay. Similarly, the extra scalar isodoublet decays into four fermions. Then, if also pair produced via the Drell-Yan mechanism, final states will have at least eight fermions. The signal may be striking due to the large number of charged leptons, but there are many open channels and may be not easy to resolve the different samples. These final states are different from those of the RR model, which will eventually allow to discriminate between both theories.

The heavy vector-like lepton doublets are also mainly produced in pairs. They violate the Glashow-Iliopoulos-Maiani mechanism [115], and can decay through a flavor changing neutral current into a light lepton and a $Z$ or Higgs boson [89]; as for sequential fermions they can also decay into a lepton and a $W$ boson through the usual charged current interaction. In these cases the final states have at least six fermions, so that the heavy leptons will 
be relatively easy to find if light enough: $M_{a} \lesssim \mathrm{TeV}$ for a $\mathrm{CM}$ energy of $14 \mathrm{TeV}$ and an integrated luminosity of $100 \mathrm{fb}^{-1}$ [89-91]. The dominant decays of these (quasi) Dirac fermions are LN conserving to a large extent.

\section{CONCLUSIONS}

To date there is no direct evidence that LN is not a symmetry of nature [1], at least at the energies and experimental sensitivities currently available. There are, however, scenarios that suggest LN may be violated at higher scale. For example, the baryon asymmetry of the universe (see for example [116]) can be explained through leptogenesis [117], yet no experimental indication of such a mechanism has been observed. Light neutrino masses and mixing angles provide the most favored explanation for neutrino oscillations, but the experiments do not distinguish between Dirac and Majorana masses, and thus, they do not require LNV; although, in many models light neutrinos are assumed to be Majorana fermions, implying that LNV is also assumed.

In contrast $0 \nu \beta \beta$ decay and appropriate signals at LHC will be sensitive to LNV effects; and in fact, these are the only way known to experimentally establish the presence of LNV. Hence the relevance of these two types of experiments. A new generation of $0 \nu \beta \beta$ decay experiments are underway (see [11, 12] for recent reviews), where a positive signal would provide conclusive evidence of LNV, and would open a new era of experimental searches and theoretical studies aiming at isolating the type of NP that mediates such a process.

The effective Lagrangian approach allows to address this theoretical question with generality and has been the subject of the above discussion. As described in the text, and detailed in the appendix, we have constructed all gauge-invariant effective operators of dimension $\leq 9$, violating LN by 2 units and involving two SM leptons (but no quarks) and any number of Higgs isodoublets and covariant derivatives (the appendix also lists LNV operators with more than 2 leptons). For each of the three possible scenarios; (i) with two LH leptons (LL), (ii) one LH lepton and one RH electron (LR), and (iii) two RH electrons (RR), there is only

one lowest order effective operator, $\mathcal{O}^{(5)}, \mathcal{O}^{(7)}$ and $\mathcal{O}^{(9)}$, respectively (see Eqs. (2), (3) and (4)). They describe the largest possible contribution to $0 \nu \beta \beta$ decay for each final electron chirality assignment. We have also identified the possible new particle additions that can generate these operators at tree level (assuming that the full theory is renormalizable). 
In general, models of neutrino masses can have an origin different from that of $0 \nu \beta \beta$ decay; irrespective of that, once the NP generates any of the LNV operators, the theory will generate neutrino masses at some loop order: at tree level, at one loop or at two loops, depending on whether the operator is $\mathcal{O}^{(5)}, \mathcal{O}^{(7)}$ or $\mathcal{O}^{(9)}$, respectively. For $\mathcal{O}^{(7,9)}$ the corresponding masses are relatively suppressed by loop and light mass (generated by chirality flips) factors. In the case of $\mathcal{O}^{(5)}$ there are no such suppressions and the same parameter giving the effective neutrino electron mass $\left(m_{\nu}\right)_{e e}$ enters in the $0 \nu \beta \beta$ decay amplitude (see Eq. (12)). Thus, both pieces of data only constrain the $\left|C^{(5)}\right| / \Lambda$ ratio. In contrast in the other two cases, and since the neutrino mass scale is fixed to be around $0.1 \mathrm{eV}$, the observation of $0 \nu \beta \beta$ decay would allow to estimate the scale $\Lambda$ of NP and the corresponding effective operator coefficient $C$ for each scenario for natural theories (and perturbative couplings). Thus, leaving to experiments searching for LFV and for collider signatures of LNV mediators. To provide an existence proof we have also constructed a simple, realistic model for each scenario, explicitly calculating the neutrino masses; as expected from dimensional arguments these masses are always proportional to $1 / \Lambda$ (see Eqs. (24), (30), (32), (41) and Ref. [35]),

as argued in the introduction: Eqs. (5), (6) and (7). Then, whereas $C^{(5)}$ and its associated $\Lambda$ can vary in between eleven orders of magnitude, at least $C_{\tau \tau}^{(7)}\left(C_{\tau \tau}^{(9)}\right)$ must be at the per million (mille) level and the corresponding $\Lambda$ of the 10 (few) TeV order. Besides, any spectrum of neutrino masses within present experimental limits can be accommodated in the first two cases, but in the RR model the neutrino hierarchy must be normal and the third mixing angle $\sin ^{2} \theta_{13} \gtrsim 0.008$, in agreement with recent observations [118-122]. On the other hand, once an explicit model is at hand, we can check if it does satisfy the present LFV constraints and bounds from large colliders; this is the case for the models discussed. Although the Higgs searches now underway at LHC will stringently restrict these models with an extended scalar sector near the electroweak scale.

\section{Acknowledgements}

This work has been supported in part by the Ministry of Economy and Competitiveness, under the grant numbers FPA2006-05294, FPA2010-17915 and FPA2011-23897, by the Junta de Andalucía grants FQM 101, FQM 03048 and FQM 6552, by the "Generalitat Valenciana" grant PROMETEO/2009/128, and by the U.S. Department of Energy grant No. DE-FG03- 
94ER40837. A.A. is supported by the MICINN under the FPU program.

\section{Appendix: Effective Lagrangian description of NP}

In this appendix we provide for completeness a brief summary of the effective Lagrangian approach and describe the procedures we followed in constructing the operators discussed in the main text.

Effective theories are useful for situations where there is a scale gap: some type of heavy physics effects contribute only virtually since the available energies are well below the scale of these interactions. Technically one differentiates between the case where the underlying physics decouples [34] and when it does not. For the first case the low-energy effective theory is obtained by a formal expansion in inverse powers of the heavy scale, the existence of which is guaranteed by the decoupling theorem [34] (see also [123]). For non-decoupling heavy physics the effective theory is obtained as a derivative expansion [15].

Here we shall assume that the heavy physics is both decoupling and weakly coupled, so that a perturbative expansion is appropriate; the characteristic scale of these new interactions will be denoted by $\Lambda$. The general parameterization of NP effects using effective interactions is valid at energies below $\Lambda$. The procedure is straightforward: one constructs all Lorentz-invariant operators involving the light fields and their derivatives and respecting the low-energy local symmetries (here, the SM gauge symmetries); the effective Lagrangian is then the linear combination of all such operators, where the $\Lambda$-dependent coefficients parametrize all possible (weakly-coupled and decoupling) types of heavy physics. In our case the NP will violate LN but will not couple to quarks. If the theory underlying the SM were known then one could derive the low-energy effective theory coefficients in terms of the parameters of the model. It may then happen that some operators will be absent or may appear with suppressed coefficients due to some as yet unknown symmetry. Not knowing the correct SM extension, the effective Lagrangian coefficients are treated as unknowns susceptible to experimental determination.

The coefficient of an operator of (canonical) dimension $n$ is proportional to $\Lambda^{4-n}$ so that the larger the dimension of the operator the smaller its effect; given a finite experimental precision this implies that operators with $n$ sufficiently large can be ignored. In addition, operators that are generated by heavy particle loops have coefficients that receive a typical 
loop suppression factor $\sim 1 /(4 \pi)^{2}$. It is important to note that any operator that respects the local symmetries of the SM will be generated by the NP at some loop level. Whether this happens at tree level depends on the operator and the details of the theory underlying the SM; it is a simple exercise to determine the operators and types of NP that have this property. It is also easy to device types of NP for which all tree-level-generated operators (TLGOs) are absent ${ }^{7}$. Dominating effects are then associated with the lowest-dimension TLGOs contributing to the process at hand. In this it is important to note that whether an operator is generated at tree level or not depends on the details of the heavy physics; there are, however, operators that are necessarily loop generated by all modalities of heavy physics [125]; we call these loop-generated operators (LGOs). It is also worth keeping in mind that in most cases the effects from LGOs will compete with those generated radiatively by the SM and are often subdominant; exceptions occur when the SM effects are absent due to some accidental SM symmetry, such as custodial symmetry or LN. Except for these cases LGO effects lie beyond the experimental sensitivity of current experiments ${ }^{8}$. There is one additional observation that can be used to simplify the effective Lagrangian: if two operators $\mathcal{O}$ and $\mathcal{O}^{\prime}$ are such that the combination $\mathcal{O}-\mathcal{O}^{\prime}$ vanishes on-shell (that is, when the classical equations of motion are imposed), then the $S$-matrix depends only on the sum of the corresponding operator coefficients [126, 127], so that one of the operators can be omitted from the effective Lagrangian parameterization ${ }^{9}$.

As emphasized previously, the main feature of the processes we will be interested in is that they exhibit LNV. The operators of interest have dimensions $\geqslant 5$; many of them have been enumerated in earlier publications, including operators involving quarks [20-23], though such catalogs are not exhaustive. We do not pretend to provide a complete list of operators but concentrate instead on those low-dimensional TLGOs with two leptons and any number of bosons and derivatives that can be probed experimentally and provide leading effects for wide classes of interesting heavy physics. In the following we will assume that the low-energy

\footnotetext{
${ }^{7}$ For example, if there is a discrete symmetry under which all the SM particles are singlets but none of the new particles are [124].

8 This means that radiative effects generated by the heavy excitations are too small to be observed, it does not preclude direct observation of new particles (provided the energy available is high enough). In these cases the effective Lagrangian approach is, however, inapplicable.

${ }^{9}$ Note in particular that this result implies that the experimental sensitivity to $\mathcal{O}$ and $\mathcal{O}^{\prime}$ is the same: one cannot replace an operator $\mathcal{O}^{\prime}$ by an equivalent one $\mathcal{O}$ and find weaker limits on $\mathcal{O}$ than on $\mathcal{O}^{\prime}$.
} 
excitations are those of the SM, with a scalar sector containing $n_{d} \geq 1$ doublets $\phi_{i}, i=$ $1, \ldots, n_{d}$ of hypercharge $1 / 2$. Although generally we assumed only one (Higgs) isodoublet when classifying the possible higher-order operators, it is worth considering the case $n_{d}>1$ because this allows the presence of additional operators that are absent in the one-doublet case for symmetry reasons; we provide examples of this in the tables below. Moreover, as we illustrated in Section V 2, the introduction of extra scalar doublets may allow for simpler, phenomenologically viable, fundamental theories. In contrast, no additional light fermions are assumed to exist, in particular, any RH gauge-singlet fermions (such as RH neutrinos) are assumed to be heavy ${ }^{10}$. In presenting our expressions we will not display family indices, though in general these are present.

In order to simplify the notation through this appendix the LH lepton isodoublets are denoted by $\ell$ and RH lepton isosinglets by $e$; here we will consider operators involving only leptons, those involving quarks will be discussed in a future publication. We now provide the list of LNV operators of dimension $\leq 9$. In doing so we merely provide field content, with the understanding that all possible gauge and Lorentz contractions are to be counted (so that each of the entries represents, in general, more than one operator). Also to make the notation simpler and clarify the physical effects of the operators we find it convenient to introduce the following composite operators

$$
N_{i a}=\phi_{i}^{\dagger} \tilde{\ell}_{a}, \quad \Psi_{i a}^{\mu}=\phi_{i}^{\dagger} D^{\mu} \tilde{\ell}_{a}, \quad J_{a b}^{\mu}=\bar{\ell}_{a} D^{\mu} \tilde{\ell}_{b}, \quad \tilde{\mathcal{W}}_{i j}^{\mu}=\phi_{i}^{\dagger} D^{\mu} \tilde{\phi}_{j}
$$

where, as before,

$$
\ell=\left(\begin{array}{c}
\nu_{L} \\
e_{L}
\end{array}\right), \quad \tilde{\ell}=\epsilon C \bar{\ell}^{T}, \quad \tilde{\phi}_{i}=\epsilon \phi_{i}^{*}
$$

with $\epsilon=i \sigma_{2}$. Lower-case indices $a, b$, etc. are family indices. For the case of a single leptonic family and one scalar isodoublet with $\langle\phi\rangle=(0, v)^{T}$ the above operators become

$$
\begin{aligned}
N & =-v \nu_{L}^{c}+\cdots \\
\Psi^{\mu} & =-v\left[\left(\partial_{\mu}+\frac{i}{2} \frac{g}{c_{\mathrm{w}}} Z_{\mu}\right) \nu_{L}^{c}+\frac{i g}{\sqrt{2}} W_{\mu}^{-} e_{L}^{c}\right]+\cdots \\
J_{\mu} & =\overline{\nu_{L}}\left[\stackrel{\leftrightarrow}{\partial_{\mu}}-i g\left(c_{\mathrm{w}} Z_{\mu}+s_{\mathrm{w}} A_{\mu}\right)\right] e_{L}^{c}+\frac{i g}{\sqrt{2}}\left(W_{\mu}^{+} \overline{\nu_{L}} \nu_{L}^{c}-W_{\mu}^{-} \overline{e_{L}} e_{L}^{c}\right)+\cdots,
\end{aligned}
$$

10 Though it will not be considered in this appendix, it is straightforward to extend the light scalar sector by adding a number of light scalar gauge-singlets. 


$$
\mathcal{W}^{\mu}=-i \frac{g}{\sqrt{2}} v^{2} W^{-\mu}+\cdots
$$

where the ellipsis denote terms involving the physical scalars and we defined $\nu_{L}^{c}=C{\overline{\nu_{L}}}^{T}$; numerically $v \simeq 174 \mathrm{GeV}$.

Given an operator $\mathcal{O}$ it is straightforward to determine whether it is loop generated or whether there are models where it appears at tree level [125]. For the second case one can also determine the types of heavy excitations involved in generating $\mathcal{O}$, of which there will be in general several possibilities. Below we will also provide the tree-level diagrams and the list of heavy excitations that can generate the leading LNV operators involving two leptons (for a systematic study of the dimension 5 operator at one loop see [128]). As in the text,

in listing the heavy particles we denote by $X_{I}^{(Y)}, \Psi_{I}^{(Y)}$ and $\Phi_{I}^{(Y)}$ a heavy vector, fermion or scalar with isospin $I$ and hypercharge $Y$, respectively. When the heavy particles can be either a heavy vector or heavy scalar with the same isospin and hypercharge, we use $B_{I}^{(Y)}$ to denote both possibilities.

\section{A. LNV operators with no quarks}

All the operators below can be generated at tree level, and it is a simple exercise to determine the types of heavy physics that can do so. The number of possibilities, however, increases rapidly with the dimension of the operator so we will restrict ourselves to those with the lowest dimension operators within each group. Still it is useful to note the following: if $\mathcal{O}$ differs from $\mathcal{O}^{\prime}$ by the presence of 2 derivatives, $\mathcal{O} \sim D^{2} \mathcal{O}^{\prime}$, then if $\mathcal{O}$ is generated at tree level in a certain model, $\mathcal{O}^{\prime}$ will be also generated at tree level. Thus, for example, if a model generates a low-energy effective vertex $\sim e_{L}^{2} W^{2}$ (from $D^{2} \ell^{2} \phi^{2}$ ), it will also generate a Majorana-mass term $\nu_{L}^{2}$ at tree level. In general, however, it will not generate a $e_{R}^{2} W^{2}$ vertex at tree level. As mentioned repeatedly, this particular example is of interest when studying $0 \nu \beta \beta$ decay.

Below we list the LNV operators of dimension $\leq 9$ not involving quarks together with the sets of heavy excitations that may generate them at tree level. The operators involving two leptons can be grouped in 3 sets according to the chirality of the light leptons (operators with $>2$ leptons will be listed at the end, and are provided for completeness only): 
a. Two LH leptons plus bosons

\begin{tabular}{|c|l|}
\hline Dim. & Operator(s) \\
\hline 5 & $\ell^{2} \phi^{2}$ \\
7 & $\ell^{2} \phi^{2} \Omega$ \\
9 & $\ell^{2} \phi^{2} \Omega^{2}$ \\
\hline
\end{tabular}

Where $\Omega$ denotes either $D^{2}$ or $\phi \tilde{\phi}$ :

$$
\Omega \sim\left(D^{2}, \phi \tilde{\phi}\right)
$$

So that, for example, $\ell^{2} \phi^{2} \Omega$ corresponds to $\ell^{2} \phi^{3} \tilde{\phi}$ or $D^{2} \ell^{2} \phi^{2}$. As mentioned above, each entry represents a series of operators obtained by making all possible index contractions and having the derivatives operate on all fields. Considering all possible contractions the operators of dimension 5 are simply

$$
\mathcal{O}_{i j a b}^{(5) \dagger}=N_{i a}^{T} C N_{j b} .
$$

The operators of dimension 7 in this category are

$$
\begin{aligned}
\mathcal{O}_{i j a b}^{(7-\mathrm{I})} & =\Psi_{i a}^{T} \cdot C \Psi_{j b}, \\
\mathcal{O}_{i j a b}^{(7-\mathrm{II})} & =J_{a b} \cdot \tilde{\mathcal{W}}_{i j}, \\
\mathcal{O}_{i j a b}^{(7-\mathrm{III})} & =N_{i a}^{T} C \partial_{\mu} \Psi_{j b}^{\mu},
\end{aligned}
$$

as well as $\mathcal{O}_{i j a b}^{(5)}\left(\phi_{k}^{\dagger} \phi_{l}\right)$. We will not consider operators of dimension 9 in this category.

The heavy excitations that can generate (47) and (48) at tree level can be read off Table III and are listed in (33). One may think that (48) are still relevant in that they involve also the gauge bosons, but in actual calculations any amplitude involving these effective operators will have a counterpart involving (47) together with SM vertices. The second amplitude will be suppressed by only $1 / \Lambda$, compared to $1 / \Lambda^{3}$ for the first one, with the same scale in both cases. As a result all effects of (48) are subdominant in any process; accordingly, we ignore these operators in the following. The same holds for operators of the form $\mathcal{O}^{(5)}\left(\phi^{\dagger} \phi\right)$, and for operators of dimension 9 in this category.

b. One RH and one LH lepton plus bosons

\begin{tabular}{|c|l|}
\hline Dim. & Operator(s) \\
\hline 7 & $\operatorname{De\ell }^{3}$ \\
9 & $\operatorname{De\ell }^{3} \Omega$ \\
\hline
\end{tabular}




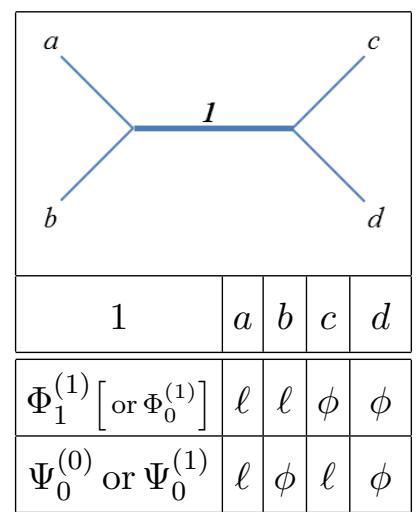

TABLE III: Diagrams generating $\mathcal{O}^{(5)}$ at tree level; fields in smaller font inside brackets correspond to models with more than one light scalar isodoublet.

The leading operator of this class has dimension 7 and is given by

$$
\mathcal{O}_{i j k a b}^{(7)}=\tilde{\mathcal{W}}_{i j}^{\mu}\left(\overline{e_{a}} \gamma^{\mu} N_{k b}\right)=i x_{i} x_{j} x_{k} m_{\mathrm{w}} v^{2} \overline{e_{a}} W^{-} P_{R} \nu_{b}^{c}+\cdots
$$

where the ellipsis denote terms involving the physical scalars and

$$
\left\langle\phi_{i}\right\rangle=v x_{i}, \quad \sum_{i} x_{i}^{2}=1, \quad m_{W}^{2}=\frac{1}{2} g^{2} v^{2}
$$

with $g$ the $S U(2)$ gauge-coupling constant.

In order to determine the types of heavy excitations that generate $\mathcal{O}^{(7)}$ we proceed as follows. We first write (omitting subindices for simplicity)

$$
\mathcal{O}^{(7)}=\left(\bar{e} \gamma^{\mu} \phi^{\dagger} \tilde{\ell}\right)\left(\phi^{\dagger} \partial_{\mu} \tilde{\phi}\right)+\cdots
$$

where the ellipsis denote terms with vector bosons. This term will be generated by graphs containing two charged leptons of opposite chiralities and 3 light scalars as external legs. A model that generates this graph at tree level must also generate the full operator (at tree level) due to gauge invariance. In practice this is obtained by attaching an appropriate number of light gauge boson lines to the internal heavy propagators at all points allowed by the quantum numbers. The graphs that can generate the term (52) at tree level can have two topologies, but chirality prevents one of them; the remaining tree-level graphs are given in Table IV and listed in (34). 


\begin{tabular}{|c|c|c|c|c|c|c|}
\hline 1 & 2 & $a$ & $b$ & $c$ & $d$ & $e$ \\
\hline$X_{1 / 2}^{(3 / 2)}$ & $X_{0}^{(1)}$ or $X_{1}^{(1)}$ & $\ell$ & $e$ & $\phi$ & $\phi$ & $\phi$ \\
\hline$X_{1 / 2}^{(3 / 2)}$ & $\Phi_{1}^{(1)}\left[\right.$ or $\left.\Phi_{0}^{(1)}\right]$ & $\ell$ & $e$ & $\phi$ & $\phi$ & $\phi$ \\
\hline$\Psi_{1}^{(0)}$ & $X_{1}^{(1)}$ & $\ell$ & $\phi$ & $e$ & $\phi$ & $\phi$ \\
\hline$\Psi_{1}^{(0)}\left[\right.$ or $\left.\Psi_{0}^{(0)}\right]$ & $\Phi_{1}^{(1)}\left[\operatorname{or} \Phi_{0}^{(1)}\right]$ & $\ell$ & $\phi$ & $e$ & $\phi$ & $\phi$ \\
\hline$\Psi_{0}^{(0)}$ & $X_{0}^{(1)}$ & $\ell$ & $\phi$ & $e$ & $\phi$ & $\phi$ \\
\hline$\Psi_{1 / 2}^{(1 / 2)}$ & $X_{0}^{(1)}$ or $X_{1}^{(1)}$ & $e$ & $\phi$ & $\ell$ & $\phi$ & $\phi$ \\
\hline$\Psi_{1 / 2}^{(1 / 2)}$ & $\Phi_{1}^{(1)}\left[\operatorname{or} \Phi_{0}^{(1)}\right]$ & $e$ & $\phi$ & $\ell$ & $\phi$ & $\phi$ \\
\hline$\Psi_{1 / 2}^{(1 / 2)}$ & $\Psi_{0}^{(0)}$ or $\Psi_{1}^{(0)}$ & $e$ & $\phi$ & $\phi$ & $\phi$ & $\ell$ \\
\hline
\end{tabular}

TABLE IV: Diagrams generating $\mathcal{O}^{(7)}$ at tree level; fields in smaller font inside brackets correspond to models with more than one light scalar isodoublet.

c. Two RH leptons plus bosons

\begin{tabular}{|c|l|}
\hline Dim. & Operator $(\mathrm{s})$ \\
\hline 7 & $e^{2} \phi^{4}$ \\
9 & $e^{2} \phi^{4} \Omega$ \\
\hline
\end{tabular}

The operators $e^{2} \phi^{4}$ and $e^{2} \phi^{5} \tilde{\phi}$ vanish for the case of a single scalar doublet. When there are more scalar isodoublets these operators generate vertices of the form $e \mathrm{H}^{+} \mathrm{H}^{+}$multiplied by neutral scalars and/or vacuum expectation values. Barring the presence of light singlecharged scalars the leading operator of this class then has dimension 9 and is given by

$$
\mathcal{O}_{i j k l a b}^{(9)}=\bar{e}_{a} e_{b}^{c} \tilde{\mathcal{W}}_{i j} \cdot \tilde{\mathcal{W}}_{k l}
$$

The tree-level graphs that can generate this operator are obtained in the same way as above. We expand

$$
\mathcal{O}^{(9)}=\left(\bar{e} e^{c}\right)\left(\phi^{\dagger} \partial^{\mu} \tilde{\phi}\right)\left(\phi^{\dagger} \partial_{\mu} \tilde{\phi}\right)+\cdots,
$$

where the ellipsis denote terms with vector bosons and then look for graphs with two RH electrons and 4 scalars in the external lines, which generate this term in $\mathcal{O}^{(9)}$; the rest of 


\begin{tabular}{|c|c|c|c|c|c|c|c|c|}
\hline 1 & 2 & 3 & $a$ & $b$ & $c$ & $d$ & $e$ & $f$ \\
\hline$\Phi_{0}^{(2)}$ & $B_{1 / 2}^{(3 / 2)}$ & $\Phi_{1}^{(1)}\left[\operatorname{or} \Phi_{0}^{(1)}\right]$ & $e$ & $e$ & $\phi$ & $\phi$ & $\phi$ & $\phi$ \\
\hline$\Phi_{0}^{(2)}$ & $B_{1 / 2}^{(3 / 2)}$ & $X_{1}^{(1)}$ or $X_{0}^{(1)}$ & $e$ & $e$ & $\phi$ & $\phi$ & $\phi$ & $\phi$ \\
\hline$\Psi_{1 / 2}^{(1 / 2)}$ & $B_{1 / 2}^{(3 / 2)}$ & $\Phi_{1}^{(1)}\left[\operatorname{or} \Phi_{0}^{(1)}\right]$ & $e$ & $\phi$ & $e$ & $\phi$ & $\phi$ & $\phi$ \\
\hline$\Psi_{1 / 2}^{(1 / 2)}$ & $B_{1 / 2}^{(3 / 2)}$ & $X_{1}^{(1)}$ or $X_{0}^{(1)}$ & $e$ & $\phi$ & $e$ & $\phi$ & $\phi$ & $\phi$ \\
\hline$\Psi_{1 / 2}^{(1 / 2)}$ & $\Psi_{1}^{(0)}\left[\right.$ or $\left.\Psi_{0}^{(0)}\right]$ & $\Phi_{1}^{(1)}\left[\operatorname{or} \Phi_{0}^{(1)}\right]$ & $e$ & $\phi$ & $\phi$ & $e$ & $\phi$ & $\phi$ \\
\hline$\Psi_{1 / 2}^{(1 / 2)}$ & $\Psi_{1}^{(0)}$ & $X_{1}^{(1)}$ & $e$ & $\phi$ & $\phi$ & $e$ & $\phi$ & $\phi$ \\
\hline$\Psi_{1 / 2}^{(1 / 2)}$ & $\Psi_{0}^{(0)}$ & $X_{0}^{(1)}$ & $e$ & $\phi$ & $\phi$ & $e$ & $\phi$ & $\phi$ \\
\hline$\Psi_{1 / 2}^{(1 / 2)}$ & $\Psi_{0}^{(0)}$ or $\Psi_{1}^{(0)}$ & $\Psi_{1 / 2}^{(1 / 2)}$ & $e$ & $\phi$ & $\phi$ & $e$ & $\phi$ & $\phi$ \\
\hline$B_{1}^{(1)}$ & $\Psi_{1}^{(0)}\left[\right.$ or $\left.\Psi_{0}^{(0)}\right]$ & $\Phi_{1}^{(1)}\left[\operatorname{or} \Phi_{0}^{(1)}\right]$ & $\phi$ & $\phi$ & $e$ & $e$ & $\phi$ & $\phi$ \\
\hline$X_{1}^{(1)}$ & $\Psi_{1}^{(0)}$ & $X_{1}^{(1)}$ & $\phi$ & $\phi$ & $e$ & $e$ & $\phi$ & $\phi$ \\
\hline$X_{0}^{(1)}$ & $\Psi_{0}^{(0)}$ & $X_{0}^{(1)}$ & $\phi$ & $\phi$ & $e$ & $e$ & $\phi$ & $\phi$ \\
\hline
\end{tabular}

TABLE V: Diagrams generating $\mathcal{O}^{(9)}$ at tree level with three virtual particles; fields in smaller font inside brackets correspond to models with more than one light scalar isodoublet.

the operator will necessarily be generated because we assume the underlying theory respects the SM gauge symmetry. There are three diagram topologies presented in Tables V, VI and VII. The sets of heavy excitations that can generate the dimension 9 operators at tree level are listed in (35).

d. More than 2 leptons and bosons

\begin{tabular}{|c|c|c|c|}
\hline Dim. & \multicolumn{3}{|c|}{ Operator(s) } \\
\hline 7 & $\ell^{3} e^{c} \phi$ & & \\
\hline 9 & $\ell^{3} e^{c} \phi \Omega$ & $\left(D \ell^{2} \phi^{2}, \ell e \phi^{3}\right) \times\left(\ell \tilde{\ell}, e e^{c}\right)$ & $\ell^{4}\left(e^{c}\right)^{2}$ \\
\hline
\end{tabular}

where the middle term in the last line represents objects of the form $D \ell^{3} \tilde{\ell} \phi^{2}, \ell^{2} \tilde{\ell} e \phi^{3}$, etc.

The important conclusion of the above arguments is that there are only three interesting LNV operators of lowest dimension: $\mathcal{O}^{(5)}, \mathcal{O}^{(7)}, \mathcal{O}^{(9)}$. Aside form their LNV effects these 


\begin{tabular}{|c|c|c|c|c|c|c|c|c|}
\hline 1 & 2 & 3 & $a$ & $b$ & $c$ & $d$ & $e$ & $f$ \\
\hline$\Phi_{0}^{(2)}$ & $\Phi_{1}^{(1)}\left[\operatorname{or} \Phi_{0}^{(1)}\right]$ & $\Phi_{1}^{(1)}\left[\right.$ or $\left.\Phi_{0}^{(1)}\right]$ & $e$ & $e$ & $\phi$ & $\phi$ & $\phi$ & $\phi$ \\
\hline$\Phi_{0}^{(2)}$ & $\Phi_{1}^{(1)}\left[\operatorname{or} \Phi_{0}^{(1)}\right]$ & $X_{1}^{(1)}\left[\right.$ or $\left.X_{0}^{(1)}\right]$ & $e$ & $e$ & $\phi$ & $\phi$ & $\phi$ & $\phi$ \\
\hline$\Phi_{0}^{(2)}$ & $X_{1}^{(1)}$ & $X_{1}^{(1)}$ & $e$ & $e$ & $\phi$ & $\phi$ & $\phi$ & $\phi$ \\
\hline$\Phi_{0}^{(2)}$ & $X_{0}^{(1)}$ & $X_{0}^{(1)}$ & $e$ & $e$ & $\phi$ & $\phi$ & $\phi$ & $\phi$ \\
\hline$\Psi_{1 / 2}^{(1 / 2)}$ & $\Phi_{1}^{(1)}\left[\right.$ or $\left.\Phi_{0}^{(1)}\right]$ & $\Psi_{1 / 2}^{(1 / 2)}$ & $e$ & $\phi$ & $\phi$ & $\phi$ & $\phi$ & $e$ \\
\hline$\Psi_{1 / 2}^{(1 / 2)}$ & $X_{1}^{(1)}$ or $X_{0}^{(1)}$ & $\Psi_{1 / 2}^{(1 / 2)}$ & $e$ & $\phi$ & $\phi$ & $\phi$ & $\phi$ & $e$ \\
\hline
\end{tabular}

TABLE VI: As table V but with a different topology.

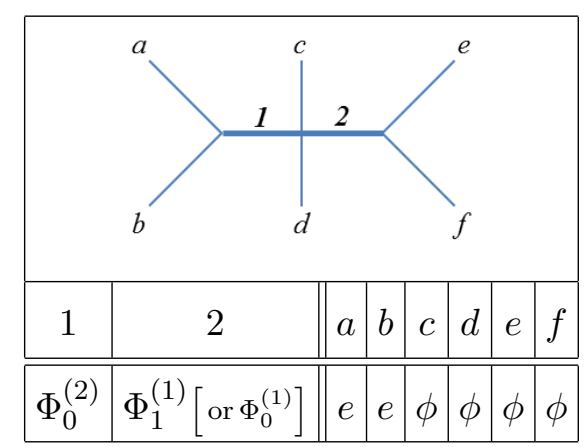

TABLE VII: As table V but with only two virtual particles.

operators in general transform non-trivially under CP.

[1] Particle Data Group Collaboration, K. Nakamura et al., Review of particle physics, J.Phys.G G37 (2010) 075021 [InSPIRE].

[2] R. Mohapatra, S. Antusch, K. Babu, G. Barenboim, M.-C. Chen, et al., Theory of neutrinos: A White paper, Rept.Prog.Phys. 70 (2007) 1757-1867, [arXiv:hep-ph/0510213] [InSPIRE]. 
[3] M. Gonzalez-Garcia and M. Maltoni, Phenomenology with Massive Neutrinos, Phys.Rept. 460 (2008) 1-129, [arXiv:0704.1800] [InSPIRE].

[4] B. Pontecorvo, Neutrino Experiments and the Problem of Conservation of Leptonic Charge, Sov.Phys.JETP 26 (1968) 984-988 [InSPIRE].

[5] Z. Maki, M. Nakagawa, and S. Sakata, Remarks on the unified model of elementary particles, Prog.Theor.Phys. 28 (1962) 870-880 [InSPIRE].

[6] T. Schwetz, M. Tortola, and J. Valle, Where we are on $\theta_{13}$ : addendum to 'Global neutrino data and recent reactor fluxes: status of three-flavour oscillation parameters', New J.Phys. 13 (2011) 109401, [arXiv: 1108.1376] [InSPIRE].

[7] R. Mohapatra and P. Pal, Massive neutrinos in physics and astrophysics, World Sci.Lect.Notes Phys. 60 (1998) 1-397 [InSPIRE].

[8] G. Racah, On the symmetry of particle and antiparticle, Nuovo Cim. 14 (1937) 322-328 [InSPIRE].

[9] W. Furry, On transition probabilities in double beta-disintegration, Phys.Rev. 56 (1939) 1184-1193 [InSPIRE].

[10] J. Vergados, The Neutrinoless double beta decay from a modern perspective, Phys.Rept. 361 (2002) 1-56, [arXiv:hep-ph/0209347] [InSPIRE].

[11] I. Avignone, Frank T., S. R. Elliott, and J. Engel, Double Beta Decay, Majorana Neutrinos, and Neutrino Mass, Rev.Mod.Phys. 80 (2008) 481-516, [arXiv:0708.1033] [InSPIRE].

[12] A. Barabash, Double beta decay experiments, Phys.Part.Nucl. 42 (2011) 613-627, [arXiv:1107.5663] [InSPIRE].

[13] S. R. Elliott, Recent Progress in Double Beta Decay, Mod.Phys.Lett. A27 (2012) 1230009, [arXiv:1203.1070] [InSPIRE].

[14] S. R. Coleman, J. Wess, and B. Zumino, Structure of phenomenological Lagrangians. 1., Phys.Rev. 177 (1969) 2239-2247 [InSPIRE].

[15] S. Weinberg, Phenomenological Lagrangians, Physica A96 (1979) 327 [InSPIRE]. Festschrift honoring Julian Schwinger on his 60th birthday.

[16] S. Weinberg, Effective Gauge Theories, Phys.Lett. B91 (1980) 51 [InSPIRE].

[17] J. Polchinski, Renormalization and Effective Lagrangians, Nucl.Phys. B231 (1984) 269-295 [InSPIRE].

[18] H. Georgi, Effective field theory, Ann.Rev.Nucl.Part.Sci. 43 (1993) 209-252 [InSPIRE]. 
[19] J. Wudka, Electroweak effective Lagrangians, Int.J.Mod.Phys. A9 (1994) 2301-2362, [arXiv:hep-ph/9406205] [InSPIRE].

[20] K. Babu and C. N. Leung, Classification of effective neutrino mass operators, Nucl.Phys. B619 (2001) 667-689, [arXiv:hep-ph/0106054] [InSPIRE].

[21] K. Choi, K. S. Jeong, and W. Y. Song, Operator analysis of neutrinoless double beta decay, Phys.Rev. D66 (2002) 093007, [arXiv:hep-ph/0207180] [InSPIRE].

[22] J. Engel and P. Vogel, Effective operators for double beta decay, Phys.Rev. C69 (2004) 034304, [arXiv:nucl-th/0311072] [InSPIRE].

[23] A. de Gouvea and J. Jenkins, A Survey of Lepton Number Violation Via Effective Operators, Phys. Rev. D77 (2008) 013008, [arXiv:0708.1344] [InSPIRE].

[24] K. Babu and J. Julio, Two-Loop Neutrino Mass Generation through Leptoquarks, Nucl.Phys. B841 (2010) 130-156, [arXiv:1006.1092] [InSPIRE].

[25] M. Duerr, M. Lindner, and A. Merle, On the Quantitative Impact of the Schechter-Valle Theorem, JHEP 1106 (2011) 091, [arXiv:1105.0901] [InSPIRE].

[26] K. Babu and J. Julio, Radiative Neutrino Mass Generation through Vector-like Quarks, Phys.Rev. D85 (2012) 073005, [arXiv:1112.5452] [InSPIRE].

[27] P. Minkowski, $\mu \rightarrow$ er at a Rate of One Out of 1-Billion Muon Decays?, Phys.Lett. B67 (1977) 421 [InSPIRE].

[28] M. Gell-Mann, P. Ramond, and R. Slansky, Complex Spinors and Unified Theories, Conf.Proc. C790927 (1979) 315-321 [InSPIRE]. Published in Supergravity, P. van Nieuwenhuizen and D.Z. Freedman (eds.), North Holland Publ. Co., 1979.

[29] T. Yanagida, Horizontal Symmetry and Masses of Neutrinos, Conf.Proc. C7902131 (1979) 95 [InSPIRE].

[30] R. N. Mohapatra and G. Senjanovic, Neutrino Mass and Spontaneous Parity Violation, Phys.Rev.Lett. 44 (1980) 912 [InSPIRE].

[31] V. Tello, M. Nemevsek, F. Nesti, G. Senjanovic, and F. Vissani, Left-Right Symmetry: from LHC to Neutrinoless Double Beta Decay, Phys.Rev.Lett. 106 (2011) 151801, [arXiv:1011.3522] [InSPIRE].

[32] S. Weinberg, Baryon and Lepton Nonconserving Processes, Phys.Rev.Lett. 43 (1979) 1566-1570 [InSPIRE].

[33] H. Weldon and A. Zee, Operator Analysis of New Physics, Nucl.Phys. B173 (1980) 269 
[InSPIRE].

[34] T. Appelquist and J. Carazzone, Infrared Singularities and Massive Fields, Phys.Rev. D11 (1975) 2856 [InSPIRE].

[35] F. del Aguila, A. Aparici, S. Bhattacharya, A. Santamaria, and J. Wudka, A realistic model of neutrino masses with a large neutrinoless double beta decay rate, JHEP 1205 (2012) 133, [arXiv:1111.6960] [InSPIRE].

[36] A. Zee, A Theory of Lepton Number Violation, Neutrino Majorana Mass, and Oscillation, Phys.Lett. B93 (1980) 389 [InSPIRE].

[37] A. Zee, Quantum numbers of Majorana neutrino masses, Nucl.Phys. B264 (1986) 99 [InSPIRE].

[38] K. Babu, Model of 'Calculable' Majorana Neutrino Masses, Phys.Lett. B203 (1988) 132 [InSPIRE].

[39] E. Ma, Verifiable radiative seesaw mechanism of neutrino mass and dark matter, Phys.Rev. D73 (2006) 077301, [arXiv:hep-ph/0601225] [InSPIRE].

[40] H. Klapdor-Kleingrothaus, A. Dietz, L. Baudis, G. Heusser, I. Krivosheina, et al., Latest results from the Heidelberg-Moscow double beta decay experiment, Eur.Phys.J. A12 (2001) 147-154, [arXiv:hep-ph/0103062] [InSPIRE].

[41] IGEX Collaboration, C. Aalseth et al., The IGEX Ge-76 neutrinoless double beta decay experiment: Prospects for next generation experiments, Phys.Rev. D65 (2002) 092007, [arXiv:hep-ex/0202026] [InSPIRE].

[42] K. Muto, E. Bender, and H. Klapdor, Nuclear Structure Effects on the Neutrinoless Double Beta Decay, Z.Phys. A334 (1989) 187-194 [InSPIRE].

[43] H. Pas, M. Hirsch, H. Klapdor-Kleingrothaus, and S. Kovalenko, Towards a superformula for neutrinoless double beta decay, Phys.Lett. B453 (1999) 194-198 [InSPIRE].

[44] H. Pas, M. Hirsch, H. Klapdor-Kleingrothaus, and S. Kovalenko, A Superformula for neutrinoless double beta decay. 2. The Short range part, Phys.Lett. B498 (2001) 35-39, [arXiv:hep-ph/0008182] [InSPIRE].

[45] E. Ma, Naturally small seesaw neutrino mass with no new physics beyond the TeV scale, Phys.Rev.Lett. 86 (2001) 2502-2504, [arXiv:hep-ph/0011121] [InSPIRE].

[46] M. Aoki, S. Kanemura, and O. Seto, Neutrino mass, Dark Matter and Baryon Asymmetry via TeV-Scale Physics without Fine-Tuning, Phys.Rev.Lett. 102 (2009) 051805, 
[arXiv:0807.0361] [InSPIRE].

[47] W. Chao, Neutrino Masses and A TeV Scale Seesaw Mechanism, Phys.Rev. D82 (2010) 016008, [arXiv:1003.1468] [InSPIRE].

[48] A. Ibarra, E. Molinaro, and S. Petcov, TeV Scale See-Saw Mechanisms of Neutrino Mass Generation, the Majorana Nature of the Heavy Singlet Neutrinos and $(\beta \beta)_{0 \nu}$-Decay, JHEP 1009 (2010) 108, [arXiv: 1007.2378] [InSPIRE].

[49] M.-C. Chen and J. Huang, TeV Scale Models of Neutrino Masses and Their Phenomenology, Mod.Phys.Lett. A26 (2011) 1147-1167, [arXiv:1105.3188] [InSPIRE].

[50] J. F. Oliver and A. Santamaria, Neutrino masses from operator mixing, Phys.Rev. D65 (2002) 033003, [arXiv:hep-ph/0108020] [InSPIRE].

[51] C.-S. Chen, C. Geng, and J. Ng, Unconventional Neutrino Mass Generation, Neutrinoless Double Beta Decays, and Collider Phenomenology, Phys.Rev. D75 (2007) 053004, [arXiv:hep-ph/0610118] [InSPIRE].

[52] C.-S. Chen, C.-Q. Geng, J. N. Ng, and J. M. Wu, Testing radiative neutrino mass generation at the LHC, JHEP 0708 (2007) 022, [arXiv:0706.1964] [InSPIRE].

[53] W. Konetschny and W. Kummer, Nonconservation of Total Lepton Number with Scalar Bosons, Phys.Lett. B70 (1977) 433 [InSPIRE].

[54] T. Cheng and L.-F. Li, Neutrino Masses, Mixings and Oscillations in SU(2) x U(1) Models of Electroweak Interactions, Phys.Rev. D22 (1980) 2860 [InSPIRE].

[55] G. Lazarides, Q. Shafi, and C. Wetterich, Proton Lifetime and Fermion Masses in an SO(10) Model, Nucl.Phys. B181 (1981) 287 [InSPIRE].

[56] M. Magg and C. Wetterich, Neutrino Mass Problem and Gauge Hierarchy, Phys.Lett. B94 (1980) 61 [InSPIRE].

[57] J. Schechter and J. Valle, Neutrino Masses in SU(2) x U(1) Theories, Phys.Rev. D22 (1980) 2227 [InSPIRE].

[58] R. N. Mohapatra and G. Senjanovic, Neutrino Masses and Mixings in Gauge Models with Spontaneous Parity Violation, Phys.Rev. D23 (1981) 165 [InSPIRE].

[59] R. Foot, H. Lew, X. He, and G. C. Joshi, Seesaw Neutrino Masses Induced by a Triplet of Leptons, Z.Phys. C44 (1989) 441 [InSPIRE].

[60] E. Ma and D. Roy, Heavy triplet leptons and new gauge boson, Nucl.Phys. B644 (2002) 290-302, [arXiv:hep-ph/0206150] [InSPIRE]. 
[61] S. King, Neutrino mass models, Rept.Prog.Phys. 67 (2004) 107-158, [arXiv:hep-ph/0310204] [InSPIRE].

[62] G. Altarelli and F. Feruglio, Models of neutrino masses and mixings, New J.Phys. 6 (2004) 106, [arXiv:hep-ph/0405048] [InSPIRE]. Submitted to the focus Issue on 'Neutrino Physics' edited by F. Halzen, M. Lindner and A. Suzuki.

[63] A. Ibarra, E. Molinaro, and S. Petcov, Low Energy Signatures of the TeV Scale See-Saw Mechanism, Phys.Rev. D84 (2011) 013005, [arXiv:1103.6217] [InSPIRE].

[64] S. M. Bilenky, C. Giunti, W. Grimus, B. Kayser, and S. Petcov, Constraints from neutrino oscillation experiments on the effective Majorana mass in neutrinoless double beta decay, Phys.Lett. B465 (1999) 193-202, [arXiv:hep-ph/9907234] [InSPIRE].

[65] S. M. Bilenky, S. Pascoli, and S. Petcov, Majorana neutrinos, neutrino mass spectrum, CP violation and neutrinoless double beta decay. 1. The Three neutrino mixing case, Phys.Rev. D64 (2001) 053010, [arXiv:hep-ph/0102265] [InSPIRE].

[66] T. Lee, A Theory of Spontaneous T Violation, Phys.Rev. D8 (1973) 1226-1239 [InSPIRE].

[67] S. Weinberg, A New Light Boson?, Phys.Rev.Lett. 40 (1978) 223-226 [InSPIRE].

[68] F. Wilczek, Problem of Strong $p$ and $t$ Invariance in the Presence of Instantons, Phys.Rev.Lett. 40 (1978) 279-282 [InSPIRE].

[69] N. G. Deshpande and E. Ma, Pattern of Symmetry Breaking with Two Higgs Doublets, Phys.Rev. D18 (1978) 2574 [InSPIRE].

[70] H. Haber, G. L. Kane, and T. Sterling, The Fermion Mass Scale and Possible Effects of Higgs Bosons on Experimental Observables, Nucl.Phys. B161 (1979) 493 [InSPIRE].

[71] J. F. Donoghue and L. F. Li, Properties of Charged Higgs Bosons, Phys.Rev. D19 (1979) 945 [InSPIRE].

[72] J. F. Gunion, H. E. Haber, G. L. Kane, and S. Dawson, The Higgs Hunter's Guide, Front.Phys. 80 (2000) 1-448 [InSPIRE].

[73] G. Branco, P. Ferreira, L. Lavoura, M. Rebelo, M. Sher, et al., Theory and phenomenology of two-Higgs-doublet models, Phys.Rept. 516 (2012) 1-102, [arXiv:1106.0034] [InSPIRE].

[74] F. del Aguila, J. Aguilar-Saavedra, J. . de Blas, and M. Perez-Victoria, Electroweak constraints on see-saw messengers and their implications for $L H C$, [arXiv:0806.1023] [InSPIRE]. Published in the Proceedings of "43rd Rencontres de Moriond on Electroweak Interactions and Unified Theories", 1-8 Mar 2008, La Thuile, Italy. 
[75] S. Kanemura and K. Yagyu, Radiative corrections to electroweak parameters in the Higgs triplet model and implication with the recent Higgs boson searches, [arXiv:1201.6287] [InSPIRE].

[76] F. del Aguila and M. J. Bowick, Suppression of Lepton Number Violation Mediated By Delta I = 0 Mass Fermions, Phys.Lett. B119 (1982) 144 [InSPIRE].

[77] P. Langacker and D. London, Mixing Between Ordinary and Exotic Fermions, Phys.Rev. D38 (1988) 886 [InSPIRE].

[78] E. Nardi, E. Roulet, and D. Tommasini, Global analysis of fermion mixing with exotics, Nucl.Phys. B386 (1992) 239-266 [InSPIRE].

[79] M. Blanke, A. J. Buras, B. Duling, A. Poschenrieder, and C. Tarantino, Charged Lepton Flavour Violation and (g-2)(mu) in the Littlest Higgs Model with T-Parity: A Clear Distinction from Supersymmetry, JHEP 0705 (2007) 013, [arXiv:hep-ph/0702136] [InSPIRE].

[80] F. del Aguila, J. Illana, and M. Jenkins, Precise limits from lepton flavour violating processes on the Littlest Higgs model with T-parity, JHEP 0901 (2009) 080, [arXiv: 0811.2891] [InSPIRE].

[81] F. del Aguila, J. Illana, and M. Jenkins, Muon to electron conversion in the Littlest Higgs model with T-parity, JHEP 1009 (2010) 040, [arXiv:1006.5914] [InSPIRE].

[82] T. Goto, Y. Okada, and Y. Yamamoto, Tau and muon lepton flavor violations in the littlest Higgs model with T-parity, Phys.Rev. D83 (2011) 053011, [arXiv:1012.4385] [InSPIRE].

[83] S. J. Huber and Q. Shafi, Neutrino oscillations and rare processes in models with a small extra dimension, Phys.Lett. B512 (2001) 365-372, [arXiv:hep-ph/0104293] [InSPIRE].

[84] M. Carena, A. D. Medina, N. R. Shah, and C. E. Wagner, Gauge-Higgs Unification, Neutrino Masses and Dark Matter in Warped Extra Dimensions, Phys.Rev. D79 (2009) 096010, [arXiv:0901.0609] [InSPIRE].

[85] F. del Aguila, A. Carmona, and J. Santiago, Neutrino Masses from an A4 Symmetry in Holographic Composite Higgs Models, JHEP 1008 (2010) 127, [arXiv:1001.5151] [InSPIRE].

[86] M. Raidal, A. van der Schaaf, I. Bigi, M. Mangano, Y. K. Semertzidis, et al., Flavour physics of leptons and dipole moments, Eur.Phys.J. C57 (2008) 13-182, [arXiv:0801.1826] [InSPIRE]. 
[87] T. Feldmann, Lepton Flavour Violation Theory, PoS BEAUTY2011 (2011) 017, [arXiv:1105.2139] [InSPIRE].

[88] F. del Aguila, J. de Blas, and M. Perez-Victoria, Effects of new leptons in Electroweak Precision Data, Phys.Rev. D78 (2008) 013010, [arXiv:0803.4008] [InSPIRE].

[89] F. del Aguila, L. Ametller, G. L. Kane, and J. Vidal, Vector-Like Fermion and Standard Higgs Production at Hadron Colliders, Nucl.Phys. B334 (1990) 1 [InSPIRE].

[90] J. Aguilar-Saavedra, Heavy lepton pair production at LHC: Model discrimination with multi-lepton signals, Nucl.Phys. B828 (2010) 289-316, [arXiv:0905.2221] [InSPIRE].

[91] F. del Aguila, A. Carmona, and J. Santiago, Tau Custodian searches at the LHC, Phys.Lett. B695 (2011) 449-453, [arXiv: 1007.4206] [InSPIRE].

[92] MEG Collaboration, J. Adam et al., New limit on the lepton-flavour violating decay $\mu^{+} \rightarrow e^{+} \gamma$, Phys.Rev.Lett. 107 (2011) 171801, [arXiv:1107.5547] [InSPIRE].

[93] MEG Collaboration, F. Cei, Lepton flavour violation experiments in LHC era, J.Phys.Conf.Ser. 259 (2010) 012010 [InSPIRE].

[94] W.-Y. Keung and G. Senjanovic, Majorana Neutrinos and the Production of the Right-Handed Charged Gauge Boson, Phys.Rev.Lett. 50 (1983) 1427 [InSPIRE].

[95] R. Franceschini, T. Hambye, and A. Strumia, Type-III see-saw at LHC, Phys.Rev. D78 (2008) 033002, [arXiv:0805.1613] [InSPIRE].

[96] G. Gelmini and M. Roncadelli, Left-Handed Neutrino Mass Scale and Spontaneously Broken Lepton Number, Phys.Lett. B99 (1981) 411 [InSPIRE].

[97] E. Ma and U. Sarkar, Neutrino masses and leptogenesis with heavy Higgs triplets, Phys.Rev.Lett. 80 (1998) 5716-5719, [arXiv:hep-ph/9802445] [InSPIRE].

[98] J. Gunion, J. Grifols, A. Mendez, B. Kayser, and F. I. Olness, Higgs Bosons in Left-Right Symmetric Models, Phys.Rev. D40 (1989) 1546 [InSPIRE].

[99] K. Huitu, J. Maalampi, A. Pietila, and M. Raidal, Doubly charged Higgs at LHC, Nucl.Phys. B487 (1997) 27-42, [arXiv:hep-ph/9606311] [InSPIRE].

[100] J. Gunion, C. Loomis, and K. Pitts, Searching for doubly charged Higgs bosons at future colliders, eConf C960625 (1996) LTH096, [arXiv:hep-ph/9610237] [InSPIRE].

[101] A. Akeroyd and M. Aoki, Single and pair production of doubly charged Higgs bosons at hadron colliders, Phys.Rev. D72 (2005) 035011, [arXiv:hep-ph/0506176] [InSPIRE].

[102] G. Azuelos, K. Benslama, and J. Ferland, Prospects for the search for a doubly-charged 
Higgs in the left-right symmetric model with ATLAS, J.Phys.G G32 (2006) 73-92, [arXiv:hep-ph/0503096] [InSPIRE].

[103] F. del Aguila and J. Aguilar-Saavedra, Distinguishing seesaw models at LHC with multi-lepton signals, Nucl.Phys. B813 (2009) 22-90, [arXiv:0808.2468] [InSPIRE].

[104] A. Akeroyd, C.-W. Chiang, and N. Gaur, Leptonic signatures of doubly charged Higgs boson production at the LHC, JHEP 1011 (2010) 005, [arXiv: 1009.2780] [InSPIRE].

[105] B. Dion, T. Gregoire, D. London, L. Marleau, and H. Nadeau, Bilepton production at hadron colliders, Phys.Rev. D59 (1999) 075006, [arXiv:hep-ph/9810534] [InSPIRE].

[106] F. Cuypers and S. Davidson, Bileptons: Present limits and future prospects, Eur.Phys.J. C2 (1998) 503-528, [arXiv:hep-ph/9609487] [InSPIRE].

[107] F. del Aguila, J. Aguilar-Saavedra, and J. de Blas, Trilepton signals: the golden channel for seesaw searches at LHC, Acta Phys.Polon. B40 (2009) 2901-2911, [arXiv:0910.2720] [InSPIRE].

[108] F. del Aguila, J. A. Aguilar-Saavedra, and J. de Blas, New neutrino interactions at large colliders, PoS ICHEP2010 (2010) 296, [arXiv:1012.1327] [InSPIRE].

[109] P. Nath, B. D. Nelson, H. Davoudiasl, B. Dutta, D. Feldman, et al., The Hunt for New Physics at the Large Hadron Collider, Nucl.Phys.Proc.Suppl. 200-202 (2010) 185-417, [arXiv:1001.2693] [InSPIRE].

[110] CMS Collaboration, Inclusive search for doubly charged higgs in leptonic final states at $\sqrt{s}=7$ TeV. CMS-PAS-HIG-11-007, 2011. http://cdsweb.cern.ch/record/1369542.

[111] ATLAS Collaboration, Search for Doubly Charged Higgs Boson Production in Like-sign Muon Pairs in pp Collisions at $\sqrt{s}=7$ TeV, Tech. Rep. ATLAS-CONF-2011-127, CERN, Geneva, Sep, 2011. http://cdsweb.cern.ch/record/1383792.

[112] Do Collaboration, V. M. Abazov et al., Search for doubly-charged Higgs boson pair production in $p \bar{p}$ collisions at $\sqrt{s}=1.96$ TeV, Phys.Rev.Lett. 108 (2012) 021801, [arXiv:1106.4250] [InSPIRE].

[113] CDF Collaboration, A Search for Doubly-Charged Bosons decaying to Like-Sign Dileptons at CDF with $6.1 \mathrm{fb}^{-1}$, Tech. Rep. CDF/PHYS/EXO/PUBLIC/10509, FERMILAB, Sep, 2011. http:

//www-cdf.fnal.gov/physics/exotic/r2a/20110407. samesigndileptons/hpp.html.

[114] C.-W. Chiang, T. Nomura, and K. Tsumura, Search for doubly charged Higgs bosons using 
the same-sign diboson mode at the LHC, Phys.Rev. D85 (2012) 095023, [arXiv:1202.2014] [InSPIRE].

[115] S. Glashow, J. Iliopoulos, and L. Maiani, Weak Interactions with Lepton-Hadron Symmetry, Phys.Rev. D2 (1970) 1285-1292 [InSPIRE].

[116] A. Dolgov, Baryogenesis, Nucl.Phys.Proc.Suppl. 35 (1994) 28-43, [arXiv:hep-ph/9312208] [InSPIRE].

[117] M. Fukugita and T. Yanagida, Baryogenesis Without Grand Unification, Phys.Lett. B174 (1986) 45 [InSPIRE].

[118] The MINOS Collaboration, P. Adamson et al., Measurement of the neutrino mass splitting and flavor mixing by MINOS, Phys.Rev.Lett. 106 (2011) 181801, [arXiv:1103.0340] [InSPIRE].

[119] T2K Collaboration, K. Abe et al., Indication of Electron Neutrino Appearance from an Accelerator-produced Off-axis Muon Neutrino Beam, Phys.Rev.Lett. 107 (2011) 041801, [arXiv:1106.2822] [InSPIRE].

[120] DOUBLE-CHOOZ Collaboration, Y. Abe et al., Indication for the disappearance of reactor electron antineutrinos in the Double Chooz experiment, Phys.Rev.Lett. 108 (2012) 131801, [arXiv:1112.6353] [InSPIRE].

[121] DAYA-BAY Collaboration, F. An et al., Observation of electron-antineutrino disappearance at Daya Bay, Phys.Rev.Lett. 108 (2012) 171803, [arXiv:1203.1669] [InSPIRE].

[122] RENO Collaboration, J. Ahn et al., Observation of Reactor Electron Antineutrino Disappearance in the RENO Experiment, Phys.Rev.Lett. 108 (2012) 191802, [arXiv:1204.0626] [InSPIRE].

[123] J. C. Collins, Renormalization: An Introduction to Renormalization, the Renormalization Group and the Operator-Product Expansion (Cambridge Monographs on Mathematical Physics). Cambridge University Press, 1984. [InSPIRE].

[124] J. Wudka, Conditions for evading the limits on the scale of new physics, J.Phys. G31 (2005) 1401-1412 [InSPIRE].

[125] C. Arzt, M. Einhorn, and J. Wudka, Patterns of deviation from the standard model, Nucl.Phys. B433 (1995) 41-66, [arXiv:hep-ph/9405214] [InSPIRE].

[126] H. Georgi, On-shell effective field theory, Nucl.Phys. B361 (1991) 339-350 [InSPIRE]. 
[127] C. Arzt, Reduced effective Lagrangians, Phys.Lett. B342 (1995) 189-195, [arXiv:hep-ph/9304230] [InSPIRE].

[128] F. Bonnet, M. Hirsch, T. Ota, and W. Winter, Systematic study of the $d=5$ Weinberg operator at one-loop order, [arXiv:1204.5862] [InSPIRE]. 